\title{
Algebraic model for scattering in three-s -cluster systems. I. Theoretical background
}

\author{
V. Vasilevsky, A. V. Nesterov, F. Arickx, and J. Broeckhove \\ Bogolyubov Institute for Theoretical Physics, Kiev, Ukraine \\ and Universiteit Antwerpen (RUCA), Department of Mathematics and Computer Science, Antwerp, Belgium
}

(Received 14 April 2000; published 14 February 2001)

\begin{abstract}
A framework to calculate two-particle matrix elements for fully antisymmetrized three-cluster configurations is presented. The theory is developed for a scattering situation described in terms of the algebraic model. This means that the nuclear many-particle state and its asymptotic behavior are expanded in terms of oscillator states of the intercluster coordinates. The generating function technique is used to optimize the calculation of matrix elements. In order to derive the dynamical equations, a multichannel version of the algebraic model is presented.
\end{abstract}

DOI: 10.1103/PhysRevC.63.034606

PACS number(s): 24.10.-i, 21.60.Gx, 21.60.Fw, 03.65.Nk

\section{INTRODUCTION}

Since 1980, the so-called algebraic model (AM) of the resonating group method has been used in the investigation of bound and continuum states of nuclear systems. Initially the AM was applied to binary cluster configurations [1-5]. Later on it was extended to describe binary clusters coupled to collective (quadrupole and monopole) channels [5-8]. Quite recently, three-cluster configurations were considered in the AM framework [9-11]. Such configurations play a significant role in light nuclei, in particular for reactions of astrophysical interest.

The algebraic model represents the nuclear many-particle wave functions through their expansions in harmonic oscillator eigenstates. The use of a basis of square integrable states reduces the Schrödinger equation to a matrix equation. This procedure is well-known for bound states, but is also applicable to continuum states when the appropriate boundary conditions are imposed on the expansion coefficients $[1,2,12-14]$. Thus the algebraic model provides a unified approach to bound and continuous spectra based on familiar matrix techniques.

Solving an AM scattering problem requires two major steps. The first is to define the basis states relevant to the scattering channels being investigated and to compute the Hamiltonian matrix elements in that basis. The second is to consider the description of the asymptotic region and boundary conditions in the basis and to solve the matrix equation subject to those boundary conditions.

We elaborate on both steps in this paper for a fully antisymmetrized three-cluster system, and introduce the proper three-cluster continuum boundary conditions. Such a description is still lacking in the literature. Three-cluster systems have been considered in some calculations, but there the continuum was approximated through sets of two-cluster configurations [15-17].

In our calculation the Pauli principle will be treated rigorously by taking full antisymmetrization into account for all nucleons in the system. The basis states will be defined by a composition of three $s$ clusters with "frozen" internal structure. The relative motions of the clusters are the relevant degrees of freedom. They are described by the hyperspherical harmonics method (HHM). It makes a natural formula- tion of the proper boundary conditions possible. The matrix elements of kinetic and potential energy in the three-cluster basis states are calculated using the generating function technique $[5,18,19]$, and methods for an explicit derivation will be presented.

The asymptotic behavior of relative motion of clusters is obtained by considering large intercluster distances where the interaction between clusters approaches zero. We use the folding approximation to determine the asymptotic wave functions. The folding approximation amounts to solving the scattering equations without nucleon-nucleon interaction and without intercluster antisymmetrization.

In the case of neutral clusters, i.e., when intercluster Coulomb interactions are absent, the asymptotic equations are uncoupled in the hyperangular momentum $K$ that is typical in the HHM. We will therefore introduce individual scattering channels characterized by quantum number $K$. In configurations with charged clusters the Coulomb interaction also couples $K$ channels in the asymptotic region. Again the folding approximation will be used to arrive at the appropriate asymptotic wave equations. A multiple channel approach which couples all $K$ channels has to be used to solve the full AM system of equations. This extends the theoretical foundation of the AM given in Ref. [20] for two-cluster systems.

In part II of this paper, the techniques discussed here will be applied to the three cluster configurations $\alpha+n+n$ for ${ }^{6} \mathrm{He}$, and $\alpha+p+p$ for ${ }^{6} \mathrm{Be}$.

\section{THREE-s -CLUSTER SYSTEMS}

\section{A. Wave functions}

The many-particle wave function for a three-cluster system of $A$ nucleons $\left(A=A_{1}+A_{2}+A_{3}\right)$ can be written, using the antisymmetrization operator $\mathcal{A}$, as follows:

$$
\Psi\left(\mathbf{q}_{1}, \ldots, \mathbf{q}_{A-1}\right)=\mathcal{A}\left[\Psi_{1}\left(A_{1}\right) \Psi_{2}\left(A_{2}\right) \Psi_{3}\left(A_{3}\right) \Psi_{R}(R)\right],
$$

where the center of mass of the $A$-nucleon system has been eliminated by the use of Jacobi coordinates $\mathbf{q}_{i}$. The cluster wave functions $\Psi_{i}\left(A_{i}\right)$ 


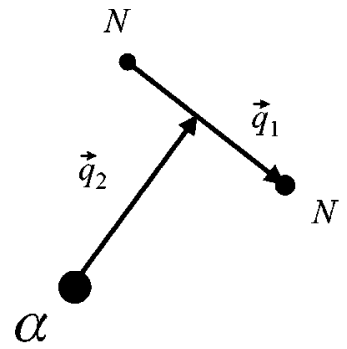

$4+2$

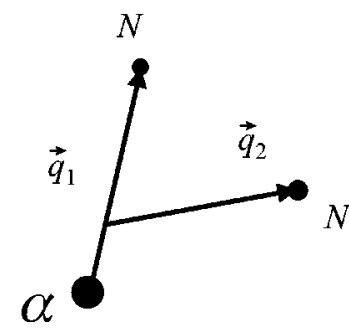

$5+1$

FIG. 1. Two configurations of the Jacobi coordinates for the three-cluster system $\alpha+N+N$.

$$
\Psi_{i}\left(A_{i}\right)=\Psi_{i}\left(\mathbf{q}_{1}^{(i)}, \ldots, \mathbf{q}_{A_{i}-1}^{(i)}\right) \quad(i=1,2,3)
$$

represent the internal structure of the $i$ th cluster, centered around its center of mass $\mathbf{R}_{i}$. In our AM study these intrinsic cluster wave functions are fixed and are Slater determinants (within a factor for the center mass motion) of harmonic oscillator $(0 s)$ states, corresponding to the ground-state configuration of the cluster $\left(A_{i} \leqslant 4\right.$ for all $\left.i\right)$. The $\Psi_{R}(R)$ wave function

$$
\Psi_{R}(R)=\Psi_{R}\left(\mathbf{q}_{1}^{(R)}, \mathbf{q}_{2}^{(R)}\right)=\Psi_{R}\left(\mathbf{q}_{1}, \mathbf{q}_{2}\right)
$$

represents the relative motion of the three clusters with respect to one another, and $\mathbf{q}_{1}$ and $\mathbf{q}_{2}$ represent Jacobi coordinates. In Fig. 1 we indicate an enumeration of possible Jacobi coordinates and their relation to the component clusters.

The state (3) is not limited to any particular type of orbital; on the contrary we will use a complete basis of harmonic oscillator states for the relative motion degrees of freedom. Thus the full $A$-particle state cannot be expressed as a single Slater determinant of single particle orbitals.

An important approximation is obtained by breaking the Pauli principle between the individual clusters, but retaining a proper quantum-mechanical description of the clusters, which is described by the wave function

$$
\Psi_{F}\left(\mathbf{q}_{1}, \ldots, \mathbf{q}_{A-1}\right)=\Psi_{1}\left(A_{1}\right) \Psi_{2}\left(A_{2}\right) \Psi_{3}\left(A_{3}\right) \Psi_{R}(R) .
$$

Because each intrinsic cluster wave function is antisymmetric, as it is a Slater determinant apart from a factor for the center mass motion, one is indeed neglecting the intercluster antisymmetrization only. This results in what is known as the "folding" model. It has the advantage of preserving the identities of the clusters and, if the intracluster structure is kept frozen, it reduces the many-particle problem to that of the relative motion of the clusters. It should, however, be mentioned that breaking the antisymmetry will introduce spurious Pauli-forbidden states which have to be eliminated.

The folding approximation will be the natural choice for calculating the asymptotic behavior of the three-cluster system, i.e., the disintegration of the system in the three noninteracting individual clusters. This amounts to the situation that all three clusters are a sufficient distance apart and intercluster interactions are no longer in force.

The folding model is, however, also an acceptable approximation in the interaction region and can serve as comparison to the fully antisymmetrized calculations. In the folding model, the clusters interact through a local, intercluster potential called the folding potential. As in the current paper $(0 s)$ determinants $\Psi_{i}\left(A_{i}\right)$ are used to describe the internal state of the clusters, the folding potential will be easily calculated and is a sum of three terms:

$$
V^{(F)}=V^{(F)}\left(\mathbf{R}_{12}\right)+V^{(F)}\left(\mathbf{R}_{23}\right)+V^{(F)}\left(\mathbf{R}_{31}\right)
$$

where each term is simply the integral

$$
\begin{aligned}
V^{(F)}\left(\mathbf{R}_{\tau v}\right)= & \sum_{i \in A_{\tau}} \sum_{j \in A_{v}} \int d \tau_{\tau} d \tau_{v}\left|\Psi_{\tau}\left(A_{\tau}\right)\right|^{2} \\
& \times V\left(\mathbf{r}_{i}-\mathbf{r}_{j}+\mathbf{R}_{\tau v}\right)\left|\Psi_{v}\left(A_{v}\right)\right|^{2} .
\end{aligned}
$$

The coordinates $\mathbf{R}_{\tau v}$ are associated with the relative position of the clusters

$$
\mathbf{R}_{\tau v}=\frac{1}{A_{\tau}} \sum_{i \in A_{\tau}} \mathbf{r}_{i}-\frac{1}{A_{v}} \sum_{j \in A_{v}} \mathbf{r}_{j}
$$

and sum to zero; they are equivalent to the $\mathbf{q}_{1}, \mathbf{q}_{2}$ Jacobi coordinates introduced earlier. In this way the folding approximation turns the three-cluster problem into an effective three-particle problem for the relative motion coordinates.

Because the cluster states are fixed and built up of $(0 s)$ orbitals, the problem of labeling the basis states with quantum numbers relates to the intercluster wave function only. This holds true whether one uses the full antisymmetrization or the folding approximation. In a two-cluster case, the set of quantum numbers describing intercluster motion is unambiguously defined. In a three-cluster case, several schemes can be used to classify the intercluster wave function in the oscillator representation. In Refs. $[9,10]$ three distinct but equivalent schemes were considered. One of these used the quantum numbers provided by the hyperspherical harmonics method (see, for instance, Refs. [21-23]). This is the classification that we will adopt. Even within this particular scheme there are several ways to classify the basis states. We shall restrict ourselves to the so-called Zernike-Brinkman basis [24]. This corresponds to the following reduction of the unitary group $U(6)$, the symmetry group of the three-particle oscillator Hamiltonian,

$$
U(6) \supset O(6) \supset O(3) \otimes O(3) \supset O(3) .
$$

This reduction provides the quantum numbers $K$ for the hypermomentum, $n$ for the hyper-radial excitation, $l_{1}$ for the angular momentum connected with the first Jacobi vector, $l_{2}$ for the angular momentum connected with the second Jacobi vector, and $L$ and $M$ for the total angular momentum obtained from coupling the partial angular momenta $l_{1}, l_{2}$. Collectively these quantum numbers will be denoted by $\nu$, i.e., $\nu$ $=\left\{n, K,\left(l_{1} l_{2}\right) L M\right\}$ in the remainder of the text.

There are a number of relations and constraints on these quantum numbers: the total angular momentum is the vector sum of the partial angular momenta $\mathbf{l}_{1}$ and $\mathbf{l}_{2}$, i.e., $\mathbf{L}=\mathbf{l}_{1}$ 
$+\mathbf{l}_{2}$ or $\left|l_{1}-l_{2}\right| \leqslant L \leqslant l_{1}+l_{2}$; by fixing the values of $l_{1}$ and $l_{2}$, we impose restrictions on the hypermomentum $K=l_{1}+l_{2}$, $l_{1}+l_{2}+2, l_{1}+l_{2}+4, \ldots$. This condition implies that for certain values of hypermomentum $K$ the sum of partial angular momenta $l_{1}+l_{2}$ cannot exceed $K$; the partial angular momenta $l_{1}$ and $l_{2}$ define the parity of the three-cluster state by the relation $\pi=(-1)^{l_{1}+l_{2}}$; for the "normal" parity states $\pi=(-1)^{L}$ the minimal value of hypermomentum is $K_{\min }$ $=L$, whereas $K_{\min }=L+1$ for the so-called "abnormal", parity states $\pi=(-1)^{L+1}$; oscillator shells with $N$ quanta are characterized by the constraint $N=2 n+K$.

Thus for a given hyperangular and rotational configuration the quantum number $n$ ladders the oscillator shells of increasing oscillator energy.

\section{THE ALGEBRAIC MODEL}

\section{A. Asymptotic solutions in coordinate representation}

The algebraic model implements a method to solve the Schrödinger equation for quantum scattering systems, in particular for nuclear cluster systems. It is based on a matrix representation of the Schrödinger equation in terms of a square integrable basis, usually harmonic oscillator states, and boundary conditions in terms of the asymptotic behavior of the expansion coefficients of the wave function. In this paper we restrict ourselves to a presentation tailored to the treatment of three-cluster systems.

In the case of three-cluster calculations, one needs to determine a proper approximation for the wave function (3). Consider an expansion of the relative wave function

$$
\Psi_{R}\left(\mathbf{q}_{1}, \mathbf{q}_{2}\right)=\sum_{\nu} c_{\nu} \Psi_{\nu}\left(\mathbf{q}_{1}, \mathbf{q}_{2}\right)
$$

with $\nu=\left\{n, K,\left(l_{1} l_{2}\right) L M\right\}$ and $\left\{\Psi_{\nu}\right\}$ a complete basis of sixdimensional oscillator states. It covers all possible types of relative motion between the three clusters.

To obtain the asymptotic behavior of the three-cluster system, we consider the folding approximation. The assumption that antisymmetrization effects between clusters are absent in the asymptotic region is a natural one. The relative motion problem of the three clusters in the absence of a potential can then be explicitly solved in the hyperspherical harmonics method (see, for instance, Refs. [21-23]). It involves the transformation of the Jacobi coordinates $\mathbf{q}_{1}$ and $\mathbf{q}_{2}$ to the hyper-radius $\rho$ and a set of hyperangles $\Omega$. The intercluster wave function in coordinate representation is expanded in hyperspherical harmonics $H_{K}^{\nu_{0}}(\Omega)$ where $\nu_{0}$ has been chosen as a shorthand for $\left(l_{1} l_{2}\right) L M$.

In the absence of the Coulomb interaction this leads to a set of equations for the hyperradial asymptotic solutions, with the kinetic energy operator as reference Hamiltonian

$$
\left\{-\frac{\hbar^{2}}{2 m}\left[\frac{d^{2}}{d \rho^{2}}+\frac{5}{\rho} \frac{d}{d \rho}-\frac{K(K+4)}{\rho^{2}}\right]-E\right\} R_{K, \nu_{0}}(\rho)=0 .
$$

The solutions can be obtained analytically and are represented by a pair of Hänkel functions for the ingoing and outgoing solutions:

$$
R_{K, \nu_{0}}^{( \pm)}(\rho)=\left\{\begin{array}{l}
H_{K+2}^{(1)}(k \rho) / \rho^{2} \\
H_{K+2}^{(2)}(k \rho) / \rho^{2}
\end{array}\right\}
$$

where

$$
k=\sqrt{\frac{2 m E}{\hbar^{2}}}
$$

One notices that these asymptotic solutions are independent of all quantum numbers $\nu_{0}$, and are determined by the value of hypermomentum $K$ only.

When charged clusters are considered the asymptotic reference Hamiltonian consists of the kinetic energy and the Coulomb interaction:

$$
\left\{-\frac{\hbar^{2}}{2 m}\left[\frac{d^{2}}{d \rho^{2}}+\frac{5}{\rho} \frac{d}{d \rho}-\frac{\|\mathcal{K}\|}{\rho^{2}}\right]+\frac{\left\|Z_{e f f}\right\|}{\rho}-E\right\}\|\mathcal{R}(\rho)\|=0 .
$$

The matrix $\|\mathcal{K}\|$ is diagonal with matrix elements $K(K+4)$, and $\left\|Z_{e f f}\right\|$, the "effective charge," is off diagonal in $K$ and $\left(l_{1} l_{2}\right)$. Different $K$ channels are now coupled. A standard approximation for solving these equations is to decouple them by assuming that the off-diagonal matrix elements of $\left\|Z_{\text {eff }}\right\|$ are sufficiently small:

$\left\{-\frac{\hbar^{2}}{2 m}\left[\frac{d^{2}}{d \rho^{2}}+\frac{5}{\rho} \frac{d}{d \rho}-\frac{K(K+4)}{\rho^{2}}\right]+\frac{Z_{e f f}}{\rho}-E\right\} R_{K, \nu_{0}}(\rho)=0$.

The constant $Z_{\text {eff }}$ depends on $K$ and $\nu_{0}$ and all parameters of the many-body system under consideration. We will restrict ourselves to this decoupling approximation, but it is to be understood that its validity has to be checked for any specific three-cluster system.

The asymptotic solutions then become

$$
R_{K, \nu_{0}}^{( \pm)}(\rho)=\left\{\begin{array}{l}
W_{i \eta, \mu}(2 i k \rho) / \rho^{5 / 2} \\
W_{-i \eta, \mu}(-2 i k \rho) / \rho^{5 / 2}
\end{array}\right\}
$$

where $W$ is the Whittaker function, $\mu=K+2$ and $\eta$ is the well-known Sommerfeld parameter

$$
\eta=\frac{m}{\hbar^{2}} \frac{Z_{e f f}}{k}
$$

As $\eta$ is a function of $K, l_{1}$, and $l_{2}$ through the parameter $Z_{\text {eff }}$, the asymptotic solutions will now be dependent on $K$ and $\nu_{0}$.

\section{B. Asymptotic solutions in oscillator representation}

The algebraic model relies on an expansion in terms of oscillator functions, and the asymptotic behavior of the corresponding expansion coefficients $c_{\nu}$. It was conjectured 
(see, for instance, Ref. [20]) that for very large values of the oscillator quantum number $n$ the expansion coefficients for physically relevant wave functions behave like

$$
c_{n}=\langle n \mid \psi\rangle \simeq \sqrt{2} \rho_{n}^{2} \psi\left(b \rho_{n}\right),
$$

where $\rho_{n}=\sqrt{4 n+2 K+6}$ corresponds to the classical turning point, $b$ is the oscillator parameter, and $\psi$ is the hyper-radial wave function.

In the case of neutral clusters this leads after substitution of the hyper-radial asymptotic solutions to the following expansion coefficients $c_{n}^{( \pm)}$:

$$
c_{n}^{( \pm) K} \simeq \sqrt{2}\left\{\begin{array}{l}
H_{K+2}^{(1)}\left(k b \rho_{n}\right) \\
H_{K+2}^{(2)}\left(k b \rho_{n}\right)
\end{array}\right\} .
$$

This result can be obtained in an alternative way $[25,26]$ by representing the Schrödinger equation, with the kinetic energy operator $\hat{T}$ as the Hamiltonian to describe the asymptotic situation, in a (hyper-radial) oscillator representation

$$
\sum_{m=0}^{\infty}\left\langle n,\left(K, \nu_{0}\right)|\hat{T}-E| m,\left(K, \nu_{0}\right)\right\rangle c_{m}^{K, \nu_{0}}=0 .
$$

This matrix equation is of a three-diagonal form because of the properties of $\hat{T}$ and the oscillator basis. Solving for the expansion coefficients $c_{n}^{K, \nu_{0}}$ leads to a three-term recurrence relation

$$
T_{n, n-1}^{K, \nu_{0}} c_{n-1}^{K, \nu_{0}}+\left(T_{n, n}^{K, \nu_{0}}-E\right) c_{n}^{K, \nu_{0}}+T_{n, n+1}^{K, \nu_{0}} c_{n+1}^{K, \nu_{0}}=0,
$$

where

$$
T_{n, m}^{K, \nu_{0}}=\left\langle n,\left(K, \nu_{0}\right)|\hat{T}| m,\left(K, \nu_{0}\right)\right\rangle .
$$

The asymptotic solutions (i.e., for high $n$ ) of this recurrence relation are then precisely given by Eq. (17).

When the Coulomb interaction is present we again apply Eq. (16) to obtain

$$
c_{n}^{( \pm) K} \simeq \sqrt{2}\left\{\begin{array}{l}
W_{i \eta, \mu}\left(2 i k b \rho_{n}\right) / \sqrt{\rho_{n}} \\
W_{-i \eta, \mu}\left(-2 i k b \rho_{n}\right) / \sqrt{\rho_{n}}
\end{array}\right\} .
$$

In this case the oscillator representation of the Schrödinger equation is no longer of a tridiagonal form, and cannot be solved analytically for the asymptotic solutions to corroborate this result.

It should be noted that the above elaborations are valid for relatively small values of momentum $k$ and sufficiently large values of discrete hyper-radius $\rho_{n}$, or when

$$
\frac{k^{2}}{\rho_{n}^{2}} \ll 1
$$

which defines the "asymptotic regime"; it shows that for any value of $k$ one can find values for $n$ where the relation is satisfied.
As we consider an asymptotic decoupling in the $\left(K, \nu_{0}\right)$ quantum numbers, one will deal with asymptotic channels characterized by the $\left(K, \nu_{0}\right)$ values. So only in the internal (or interaction) region will states with different $K$ and $\nu_{0}$ be coupled by the short-range nuclear potential and the Coulomb potential. The three-cluster system can therefore be described by a coupled-channels approach, where the individual channels are characterized by a single $K$ value, and we will henceforth refer to these channels as " $K$ channels."

\section{Multichannel AM equations}

In the current many-channel description of the algebraic model for three-cluster systems, the channels will be characterized by the a specific value of the set of quantum numbers $K, \nu_{0}$, whereas the relative motion of clusters within the channel is connected to the oscillator index $n$. We will use $K$ henceforth as a corporate index for individual channels, and assume it represents all $K, \nu_{0}$ quantum numbers.

The Schrödinger equation can be cast in a matrix equation of the form

$$
\sum_{K^{\prime}} \sum_{m}\left\langle n, K|\hat{H}-E| m, K^{\prime}\right\rangle c_{m}^{K^{\prime}}=0
$$

We will now use a representation of the dynamical equations presented in Ref. [20]. As we will consider an $S$-matrix formulation of the problem, the expansion coefficients are rewritten as

$$
c_{n}^{K}=c_{n}^{(0) K}+\delta_{K_{i} K} c_{n}^{(-) K}-S_{K_{i} K} c_{n}^{(+) K},
$$

where, for the current channel $K$, the $c_{n}^{( \pm) K}$ are the incoming and outgoing asymptotic coefficients (valid for all $n$ ), and the matrix element $S_{K_{i} K}$ describes the coupling between the current channel $K$ and the entrance channel $K_{i}$. The $c_{n}^{(0) K}$ are referred to as "residual coefficients" [20], since they represent the deviation from the asymptotic ones, and are different from zero for small $n$ only.

As shown in Refs. [12,27,14], the $c_{n}^{( \pm) K}$ satisfy the following system of equations for a given channel $K$ :

$$
\sum_{m=0}^{\infty}\left\langle n, K\left|\hat{H}_{0}-E\right| m, K\right\rangle c_{m}^{( \pm) K}=\beta_{0}^{( \pm) K} \delta_{n, 0},
$$

$\hat{H}_{0}$ being the asymptotic reference Hamiltonian, which equals the kinetic energy operator for uncharged clusters, and the kinetic energy operator plus Coulomb interaction for charged clusters. The right-hand side features $\beta_{0}^{( \pm) K}$ which is a regularization factor to eliminate the irregular behavior of the $c_{0}^{( \pm) K}$. This factor indeed allows one to solve Eq. (25) for all values of $n$ [20], leading to a regular solution for very small $n$ and to correct solutions in the asymptotic region. The value of $\beta_{0}^{( \pm) K}$ can be obtained for both reference Hamiltonians (i.e., with or without Coulomb). The set of Eqs. (25) for the asymptotic coefficients can then be solved numerically to different degrees of approximation depending on the 
requested precision. The $c_{n}^{K( \pm)}$ have the desired asymptotic behavior [cf. Eqs. (17) and (21)].

Substitution of Eq. (24) in Eq. (23) then leads to the following system of dynamical equations for the many-channel system:

$$
\begin{aligned}
\sum_{K^{\prime}} & \sum_{m}\left\langle n, K|\hat{H}-E| m, K^{\prime}\right\rangle c_{m}^{K^{\prime}(0)} \\
& -\sum_{K^{\prime}} S_{K_{i} K^{\prime}}\left[\beta_{0}^{(+) K^{\prime}} \delta_{n, 0} \delta_{K^{\prime} K^{\prime}}+V_{n}^{K K^{\prime}(+)}\right] \\
& =-\beta_{0}^{(-) K} \delta_{n, 0} \delta_{K_{i} K}-V_{n}^{K K_{i}(-)}
\end{aligned}
$$

where the dynamical coefficients $V_{n}^{K K^{\prime}( \pm)}$, defined in Ref. [20], are given by

$$
V_{n}^{( \pm) K K^{\prime}}=\sum_{m=0}^{\infty}\left\langle n, K|\hat{V}| m, K^{\prime}\right\rangle c_{m}^{( \pm) K^{\prime}}
$$

This system of equations should be solved for the residual coefficients $c_{n}^{K(0)}$ and the $S$-matrix elements $S_{K^{\prime} K}$.

To obtain an appropriate approximation to the exact solution of Eq. (26), we consider an internal region corresponding to $n<N$ and an asymptotic region with $n \geqslant N$. The choice of $N$ is such that one can expect the residual expansion coefficients $\left\{c_{n}^{(0) K}\right\}$ to be sufficiently small in the asymptotic region. Under these assumptions Eq. (26) reduces to the following set of $N+1$ equations $(n=0 \ldots N)$ :

$$
\begin{aligned}
\sum_{K^{\prime}} & \sum_{m<N}\left\langle n, K|\hat{H}-E| m, K^{\prime}\right\rangle c_{m}^{(0) K^{\prime}} \\
& -\sum_{K^{\prime}} S_{K_{i} K^{\prime}}\left[\beta_{0}^{(+) K^{\prime}} \delta_{n, 0} \delta_{K^{\prime} K}+V_{n}^{K K^{\prime}(+)}\right] \\
& =-\beta_{0}^{(-) K} \delta_{n, 0} \delta_{K_{i} K}-V_{n}^{K K_{i}(-)} .
\end{aligned}
$$

The total number of equations for a given entrance channel $K_{i}$ amounts to $N_{c h}(N+1)$, and solving the set of equations by traditional numerical linear algebra leads to the $N_{c h} N$ residual coefficients $\left\{c_{n}^{K(0)} ; K=K_{\min } \ldots K_{\max } ; n\right.$ $=0 \ldots N-1\}$ and $N_{c h} \quad S$-matrix elements $\left\{S_{K_{i} K} ; K\right.$ $\left.=K_{\min } \ldots K_{\max }\right\}$. The set of equations has to be solved for all $N_{c h}$ entrance channels labeled by $K_{i}$.

\section{Numerical solution and convergence}

The numerical solution of the AM equations crucially depends on a proper choice of $N$, distinguishing the internal from the external region. The determining factor in this is the form of the potential energy matrix elements which, contrary to the short-range coordinate character of the potential, can be of a slowly descending nature in $n$. If this is the case, a sufficiently large value of $N$ has to be chosen.

In the case of three-cluster systems it is known from literature [28] that the potential asymptotically behaves as $1 / \rho^{3}$ in the hyper-radius, with a corresponding effect on the matrix elements. It will be shown later on that the asymptotic form of the effective potential in the current case follows this behavior. It is well known [29-31] that potentials with an asymptotic tail $1 / \rho^{3}$ dramatically change the phase shift behavior in the low energy region and that special care should be taken to get convergent results. This cannot always be obtained by merely choosing a sufficiently large value of $N$.

In Ref. [20] a numerical strategy to take care of long tails in the potential in the AM was developed. It dramatically improves the convergence of the results with significantly smaller values for $N$. We refer to Ref. [20] for further details.

\section{THE GENERATING FUNCTION METHOD}

\section{A. General principle}

In this section, the general principles for calculating matrix elements in a three-cluster basis will be explained. The two main quantities of interest are: the overlap matrix, and the Hamiltonian matrix. The former is of importance because of the proper normalization of the basis states. The latter is decomposed into the kinetic energy operator, the matrix elements of which are obtained mainly by group-theoretical considerations, the potential energy operator, which in our case will be chosen to be a semirealistic two-body interaction based on a superposition of Gaussians, and the Coulomb contribution.

In this work matrix elements for two-body Gaussian interactions will be derived. From these matrix elements of other functional forms of two-body interactions can be obtained using Gaussian transforms. This latter procedure will be followed to calculate the matrix elements of the Coulomb interaction.

The basic principle of generating functions is well-known from mathematical physics. A generating function or generator state depends on a parameter, referred to as the generating coordinate, in such a way that an expansion with respect to that parameter yields basis states as expansion terms. A familiar example is the single-particle translated Gaussian wave function

$$
\phi(\mathbf{r} \mid \mathbf{R})=\exp \left\{-\frac{1}{2} \mathbf{r}^{2}+\sqrt{2} \mathbf{R} \cdot \mathbf{r}-\frac{1}{2} \mathbf{R}^{2}\right\}
$$

with the translation parameter $\mathbf{R}$ acting as generator coordinate. The choice of parametrization of the generator coordinate influences the quantum numbers of the individual basis states that are generated. In a Cartesian parametrization $\mathbf{R}$ $=\left(R_{x}, R_{y}, R_{z}\right)$ one generates the familiar Cartesian $\phi_{n_{x}}\left(R_{x}\right) \phi_{n_{y}}\left(R_{y}\right) \phi_{n_{z}}\left(R_{z}\right)$ oscillator states. With a radial parametrization $\mathbf{R}=R \check{\mathbf{R}}$ (where the inverted hat stands for a unit vector) the expansion yields

$$
\phi(\mathbf{r} \mid \mathbf{R})=\sum_{n, l, m} \mathcal{N}_{n l} R^{2 n+l} Y_{l m}(\check{\mathbf{R}}) \phi_{n l m}(\mathbf{r}),
$$

where $\mathcal{N}_{n l}$ the is normalization factor 


$$
\mathcal{N}_{n l}=\sqrt{\frac{4 \pi \Gamma(3 / 2)}{n ! \Gamma(n+l+3 / 2) 2^{2 n+l}}} .
$$

In fact, the expression

$$
\mathcal{N}_{n l} R^{2 n+l} Y_{l m}(\check{\mathbf{R}})
$$

is an orthonormal oscillator function in Fock-Bargmann space.

An underlying mathematical connection exists between such expansions, group representation theory, and coherent state analysis $[32,33]$. In the present work we exploit the generating function principle to facilitate the computation of matrix elements. The matrix element of any operator between generating states is a function of the generating coordinates on the left and right

$$
X\left(\mathbf{R}, \mathbf{R}^{\prime}\right)=\left\langle\phi(\mathbf{r} \mid \mathbf{R})|\hat{\mathbf{X}}| \phi\left(\mathbf{r} \mid \mathbf{R}^{\prime}\right)\right\rangle .
$$

Expansion of this function will yield the matrix elements between the basis states. They can be identified in the expansion by the appropriate dependence on the generator coordinates

$$
\begin{aligned}
X\left(\mathbf{R}, \mathbf{R}^{\prime}\right)= & \sum_{n l m} \sum_{n^{\prime} l^{\prime} m^{\prime}} \mathcal{N}_{n l} \mathcal{N}_{n^{\prime} l^{\prime}} R^{2 n+l} R^{\prime\left(2 n^{\prime}+l^{\prime}\right)} \\
& \times Y_{l m}^{*}(\check{\mathbf{R}}) Y_{l^{\prime} m^{\prime}}\left(\check{\mathbf{R}}^{\prime}\right)\left\langle\phi_{n l m}(\mathbf{r})|\hat{\mathbf{X}}| \phi_{n^{\prime} l^{\prime} m^{\prime}}(\mathbf{r})\right\rangle .
\end{aligned}
$$

Of course, one is not required to expand with respect to all parameters at once. Elimination of the angular dependence first, yields a partial generating function for the radial matrix elements:

$$
\begin{gathered}
X\left(\mathbf{R}, \mathbf{R}^{\prime}\right)=\sum_{l m} \sum_{l^{\prime} m^{\prime}} Y_{l m}^{*}(\check{\mathbf{R}}) Y_{l^{\prime} m^{\prime}}\left(\check{\mathbf{R}}^{\prime}\right) X_{l m ; l^{\prime} m^{\prime}}\left(R, R^{\prime}\right) \\
X_{l m ; l^{\prime} m^{\prime}}\left(R, R^{\prime}\right)= \\
\sum_{n} \sum_{n^{\prime}} \mathcal{N}_{n l} \mathcal{N}_{n^{\prime} l^{\prime}} R^{2 n+l} R^{\prime}\left(2 n^{\prime}+l^{\prime}\right) \\
\times\left\langle\phi_{n l m}(\mathbf{r})|\hat{\mathbf{X}}| \phi_{n^{\prime} l^{\prime} m^{\prime}}(\mathbf{r})\right\rangle .
\end{gathered}
$$

Such partial generating functions will prove to be particularly useful when we apply the generating function method to the three-cluster problem with six generator coordinates (corresponding to six degrees of freedom) and an extensive set $\nu=\left\{n, K,\left(l_{1} l_{2}\right) L M\right\}$ of quantum numbers.

The calculation of the matrix elements with the generating function method is a two-step process. The first step is the calculation of the generating function for the operator involved. Usually this is accomplished with analytical techniques. The second step is the expansion of the generating function with respect to the generator coordinates. Several approaches have been used in this respect. Explicit differentiation is one of them. Using recurrence relations for the expansion terms is another one [34]. In any case, the work involved here is straightforward but extremely tedious; both approaches are best implemented using algebraic manipulation software such as MATHEMATICA or MAPLE.

In this paper we introduce a representation of the generating functions in a manageable form to obtain explicit matrix elements and their connecting recurrence relations.

\section{B. Three-cluster generator state}

The customary generator state for the intercluster basis functions is given by (in what follows we shall use small $\mathbf{q}$ for the Jacobi vectors and capital $\mathbf{Q}$ for the corresponding generating coordinates)

$$
\begin{aligned}
\Psi\left(\mathbf{q}_{1}, \mathbf{q}_{2} \mid \mathbf{Q}_{1}, \mathbf{Q}_{2}\right)= & \exp \left\{-\frac{1}{2}\left(\mathbf{q}_{1}^{2}+\mathbf{q}_{2}^{2}\right)\right. \\
& \left.+\sqrt{2}\left(\mathbf{Q}_{1} \cdot \mathbf{q}_{1}+\mathbf{Q}_{2} \cdot \mathbf{q}_{2}\right)-\frac{1}{2}\left(\mathbf{Q}_{1}^{2}+\mathbf{Q}_{2}^{2}\right)\right\} .
\end{aligned}
$$

The choice of parametrization is linked to the basis states one intends to generate. Associated with our choice of basis (Zernike-Brinkman [24]), we introduce hyperspherical coordinates. The hyper-radius and hyperangles, both for spatial coordinates and for generating parameters, are defined by

$$
\begin{gathered}
\rho=\sqrt{\mathbf{q}_{1}^{2}+\mathbf{q}_{2}^{2}}, \quad q_{1}=\rho \cos \theta, \quad q_{2}=\rho \sin \theta \\
R=\sqrt{\mathbf{Q}_{1}^{2}+\mathbf{Q}_{2}^{2}}, \quad Q_{1}=R \cos \Theta, \quad Q_{2}=R \sin \Theta .
\end{gathered}
$$

Using these, one expands the generating function (34) in hyperspherical harmonic functions:

$$
\Psi\left(\mathbf{q}_{1}, \mathbf{q}_{2} \mid \mathbf{Q}_{1}, \mathbf{Q}_{2}\right)=\sum_{\nu} \Psi_{\nu}\left(\rho, \theta, \check{\mathbf{q}}_{1}, \check{\mathbf{q}}_{2}\right) \Xi_{\nu}^{*}\left(R, \Theta, \check{\mathbf{Q}}_{1}, \check{\mathbf{Q}}_{2}\right),
$$

where the full set of quantum numbers $\nu$ (introduced previously) is involved in the summation. The oscillator basis functions are

$$
\begin{aligned}
\Psi_{\nu}\left(\rho, \theta, \check{\mathbf{q}}_{1}, \check{\mathbf{q}}_{2}\right)= & \mathcal{N}_{n, K} \rho^{K} \exp \left\{-\rho^{2} / 2\right\} L_{n}^{K+2}\left(\rho^{2}\right) \\
& \times H_{K}^{\left(l_{1} l_{2}\right) L M}\left(\theta, \check{\mathbf{q}}_{1}, \check{\mathbf{q}}_{2}\right)
\end{aligned}
$$

and the generator coordinate functions are

$$
\Xi_{\nu}\left(R, \Theta, \check{\mathbf{Q}}_{1}, \check{\mathbf{Q}}_{2}\right)=\mathcal{N}_{n, K} R^{K+2 n} H_{K}^{\left(l_{1} l_{2}\right) L M}\left(\Theta, \check{\mathbf{Q}}_{1}, \check{\mathbf{Q}}_{2}\right) .
$$

Here $H$ denotes the hyperspherical harmonic function

$$
\begin{aligned}
& H_{K}^{\left(l_{1} l_{2}\right) L M}\left(\Theta, \check{\mathbf{Q}}_{1}, \check{\mathbf{Q}}_{2}\right)= \mathcal{N}_{K}^{\left(l_{1} l_{2}\right) L M} \Phi_{K}^{\left(l_{1} l_{2}\right)}(\Theta) \\
& \times\left\{Y_{l_{1}}\left(\check{\mathbf{Q}}_{1}\right) \times Y_{l_{2}}\left(\check{\mathbf{Q}}_{2}\right)\right\}_{L M} \\
& \Phi_{K}^{\left(l_{1} l_{2}\right)}(\Theta)=(\cos \Theta)^{l_{1}}(\sin \Theta)^{l_{2}} P_{K-l_{1}-l_{2} / 2}^{l_{2}+1 / 2, l_{1}+1 / 2}(\cos 2 \Theta) .
\end{aligned}
$$


From Eq. (38), one easily deduces the procedure for selecting basis functions with fixed quantum numbers $\nu$ $=\left\{n, K,\left(l_{1} l_{2}\right) L M\right\}$. One has to differentiate the generating function $(K+2 n)$-times with respect to $R$ and then to set $R$ $=0$. After that one has to integrate over $\Theta$ with the weight $\Phi_{K}^{\left(l_{1} l_{2}\right)}$ to project onto the hypermomentum $K$; one has to integrate over unit vectors $\check{\mathbf{Q}}_{1}$ and $\check{\mathbf{Q}}_{2}$ with weights $Y_{l_{1} m_{1}}\left(\check{\mathbf{Q}}_{1}\right)$ and $Y_{l_{2} m_{2}}\left(\check{\mathbf{Q}}_{2}\right)$ to project onto partial angular momenta. The order of these operations is not important and is a matter of convenience for each specific case. However these calculations, in particular those connected with integrating over hyperangle $\Theta$, are extremely extensive and cumbersome. For this reason, we introduce a new generating function appropriate for three-cluster calculations.

We start from the function below which depends on seven generating coordinates:

$$
\begin{aligned}
\Psi\left(\mathbf{q}_{1}, \mathbf{q}_{2} \mid \epsilon, \mathbf{Q}_{1}, \mathbf{Q}_{2}\right) \\
=(1+\epsilon)^{-3} \exp \left\{-\frac{1}{2} \frac{1-\epsilon}{1+\epsilon}\left(\mathbf{q}_{1}^{2}+\mathbf{q}_{2}^{2}\right)\right. \\
\left.\quad+\frac{\sqrt{2}}{1+\epsilon}\left(\mathbf{Q}_{1} \cdot \mathbf{q}_{1}+\mathbf{Q}_{2} \cdot \mathbf{q}_{2}\right)-\frac{1}{2} \frac{1}{1+\epsilon}\left(\mathbf{Q}_{1}^{2}+\mathbf{Q}_{2}^{2}\right)\right\} .
\end{aligned}
$$

It was used previously in a different context $[7,19,35]$ to describe the coupling between monopole and two-cluster degrees of freedom. In those cases, the parameter $\mathbf{Q}$ generates basisfunctions of intercluster motion while parameter $\epsilon$ generates collective monopole excitations of the $A$-nucleon system. Here, we will modify the function somewhat and use it only for the intercluster motion. We exploit the redundancy in the set of generating parameters (seven parameters vs six degrees of freedom) and the fact that all expressions up to now are valid for complex generator coordinates also. We restrict the moduli of $\mathbf{Q}_{1}$ and $\mathbf{Q}_{2}$ and set

$$
Q_{1}=S, \quad Q_{2}=-i S
$$

When the complex conjugate version of Eq. (40) is used, e.g., in the calculation of matrix elements, Eq. (42) is also complex conjugated.

We now consider the new set of generator coordinates $\epsilon, S, \check{\mathbf{Q}}_{1}, \check{\mathbf{Q}}_{2}$, and substitute Eq. (42) in Eq. (40), where the inverted hats on $\mathbf{Q}_{1}$ and $\mathbf{Q}_{2}$ again indicate the angular components of both variables. This leads to

$$
\begin{aligned}
\Psi\left(\mathbf{q} \mid \epsilon, S, \check{\mathbf{Q}}_{1}, \check{\mathbf{Q}}_{2}\right)= & (1+\epsilon)^{-3} \exp \left\{-\frac{1}{2} \frac{1-\epsilon}{1+\epsilon}\left(\mathbf{q}_{1}^{2}+\mathbf{q}_{2}^{2}\right)\right. \\
& \left.+\frac{\sqrt{2} S}{1+\epsilon}\left(\check{\mathbf{Q}}_{1} \cdot \mathbf{q}_{1}-i \check{\mathbf{Q}}_{2} \cdot \mathbf{q}_{2}\right)\right\} \\
= & \sum_{\nu} \Psi_{\nu}\left(\rho, \theta, \check{\mathbf{q}}_{1}, \check{\mathbf{q}}_{2}\right) \Phi_{\nu}\left(\epsilon, S, \check{\mathbf{Q}}_{1}, \check{\mathbf{Q}}_{2}\right),
\end{aligned}
$$

where the weights associated with each basis function are given by

$$
\begin{aligned}
\Phi_{\nu}\left(\epsilon, S, \check{\mathbf{Q}}_{1}, \check{\mathbf{Q}}_{2}\right)= & \mathcal{N}_{K}^{\left(l_{1} l_{2}\right) L M} \mathcal{N}_{n, K}(-i)^{l_{2}} \epsilon^{n} S^{K} \\
& \times\left\{Y_{l_{1}}\left(\check{\mathbf{Q}}_{1}\right) \times Y_{l_{2}}\left(\check{\mathbf{Q}}_{2}\right)\right\}_{L M} .
\end{aligned}
$$

These are of a simpler structure and easier to use than Eq. (38) because through Eq. (42) the dependence on the hyperangular coordinate has been eliminated.

The full generating function for the matrix elements $X_{\nu, \nu^{\prime}}$ of operator $\hat{\mathbf{X}}$ now has the following general structure:

$$
\begin{aligned}
X\left(\epsilon, S, \check{\mathbf{Q}}_{1}, \check{\mathbf{Q}}_{2} ; \epsilon^{\prime}, S^{\prime}, \check{\mathbf{Q}}_{1}^{\prime}, \check{\mathbf{Q}}_{2}^{\prime}\right)= & \sum_{\nu, \nu^{\prime}}\left\langle\Psi \Psi_{\nu}\left(\rho, \theta, \check{\mathbf{q}}_{1}, \check{\mathbf{q}}_{2}\right)|\hat{\mathbf{X}}| \Psi_{\nu^{\prime}}\left(\rho, \theta, \check{\mathbf{q}}_{1}, \check{\mathbf{q}}_{2}\right)\right\rangle \Phi_{\nu}^{*}\left(\epsilon, S, \check{\mathbf{Q}}_{1}, \check{\mathbf{Q}}_{2}\right) \Phi_{\nu^{\prime}}\left(\epsilon^{\prime}, S^{\prime}, \check{\mathbf{Q}}_{1}^{\prime}, \check{\mathbf{Q}}_{2}^{\prime}\right) \\
= & \sum_{\nu, \nu^{\prime}} X_{\nu, \nu^{\prime}} \mathcal{N}_{K}^{\left(l_{1} l_{2}\right) L M} \mathcal{N}_{K^{\prime}}^{\left(l_{1}^{\prime} l_{2}^{\prime}\right) L^{\prime} M^{\prime}} \mathcal{N}_{n, K} \mathcal{N}_{n^{\prime}, K^{\prime}}(-i)^{l_{2}}(i)^{l_{2}^{\prime}} \epsilon^{n} \epsilon^{n^{\prime}} S^{K} S^{\prime K^{\prime}} \\
& \times\left\{Y_{l_{1}}^{*}\left(\check{\mathbf{Q}}_{1}\right) \times Y_{l_{2}}^{*}\left(\check{\mathbf{Q}}_{2}\right)\right\}_{L M}\left\{Y_{l_{1}^{\prime}}\left(\check{\mathbf{Q}}_{1}^{\prime}\right) \times Y_{l_{2}^{\prime}}\left(\check{\mathbf{Q}}_{2}^{\prime}\right)\right\}_{L^{\prime} M^{\prime}},
\end{aligned}
$$

where again the shorthand notation for the quantum numbers $\nu=\left(n, K,\left(l_{1} l_{2}\right) L M\right)$ and $\nu^{\prime}=\left(n^{\prime}, K^{\prime},\left(l_{1}^{\prime} l_{2}^{\prime}\right) L^{\prime} M^{\prime}\right)$ is used. As explained before, we will also consider partial generating functions which have been reduced with respect to a subset of generating coordinates and their corresponding quantum numbers. Most often, we will use a reduction with respect to the angular momentum dependence, i.e., 


$$
\begin{aligned}
X(\boldsymbol{\epsilon}, S & \left.\check{\mathbf{Q}}_{1}, \check{\mathbf{Q}}_{2} ; \epsilon^{\prime}, S^{\prime}, \check{\mathbf{Q}}_{1}^{\prime}, \check{\mathbf{Q}}_{2}^{\prime}\right) \\
= & \sum_{\left(l_{1} l_{2}\right) L M,\left(l_{1}^{\prime} l_{2}^{\prime}\right) L^{\prime} M^{\prime}} \mathcal{X}_{\left(l_{1} l_{2}\right) L M ;\left(l_{1}^{\prime} l_{2}^{\prime}\right) L^{\prime} M^{\prime}}\left(\epsilon, S, \epsilon^{\prime}, S^{\prime}\right) \\
& \times\left\{Y_{l_{1}}^{*}\left(\check{\mathbf{Q}}_{1}\right) \times Y_{l_{2}}^{*}\left(\check{\mathbf{Q}}_{2}\right)\right\}_{L M}\left\{Y_{l_{1}^{\prime}}\left(\check{\mathbf{Q}}_{1}^{\prime}\right) \times Y_{l_{2}^{\prime}}\left(\check{\mathbf{Q}}_{2}^{\prime}\right)\right\}_{L^{\prime} M^{\prime}}
\end{aligned}
$$

with the partial generating function

$$
\begin{aligned}
\mathcal{X}_{\left(l_{1} l_{2}\right) L M ;\left(l_{1}^{\prime} l_{2}^{\prime}\right) L^{\prime} M^{\prime}}\left(\epsilon, S, \epsilon^{\prime}, S^{\prime}\right) & \\
= & \sum_{n, K, n^{\prime}, K^{\prime}} X_{K, n,\left(l_{1} l_{2}\right) L M ; K^{\prime}, n^{\prime},\left(l_{1}^{\prime} l_{2}^{\prime}\right) L^{\prime} M^{\prime}} \\
& \times \mathcal{N}_{K}^{\left(l_{1} l_{2}\right) L M} \mathcal{N}_{K^{\prime}}^{\left(l_{1}^{\prime} l_{2}^{\prime}\right) L^{\prime} M^{\prime}} \mathcal{N}_{n, K} \mathcal{N}_{n^{\prime}, K^{\prime}}(-i)^{l_{2}}(i)^{l_{2}^{\prime}} \\
& \times \epsilon^{n} \epsilon^{\prime}{ }^{\prime} S^{K} S^{\prime} K^{\prime}
\end{aligned}
$$

generating matrix elements for specified $\left(l_{1} l_{2}\right) L M$ and $\left(l_{1}^{\prime} l_{2}^{\prime}\right) L^{\prime} M^{\prime}$ only.

The asymmetry in the treatment of the different quantum numbers is motivated by the methodology in which the matrix elements will be used. Indeed, all quantities in this paper are calculated in the context of the algebraic model. As explained earlier the spatial asymptotic behavior is mapped onto the asymptotic behavior of the expansion coefficients in the oscillator basis. As for fixed $K$ the $n$ quantum numbers ladder through the oscillator shells, they will be needed for sufficiently high values in order to properly describe the asymptotic region.

\section{MATRIX ELEMENTS IN THE FOLDING APPROXIMATION}

In this section we derive the generating functions for the overlap and Hamiltonian in the folding approximation, for several reasons. The folding approximation is indeed the natural representation for discussing the asymptotic behavior of the three cluster system, as the antisymmetrization between clusters vanishes at large intercluster distances. The calculation of generating functions in this approximation is also illuminating for the subsequent derivation of generating functions in a fully antisymmetrized setting, as the principles are identical, but the implementation is more complex. Finally, the folding approximation provides an interesting model to discuss the importance of antisymmetrization in the interaction region.

\section{A. Matrix elements for the overlap}

The overlap of two generating functions of the form (40) is easily obtained, and can be written as

$$
\begin{aligned}
& I\left(\epsilon, \mathbf{Q}_{1}, \mathbf{Q}_{2} ; \epsilon^{\prime}, \mathbf{Q}_{1}^{\prime}, \mathbf{Q}_{2}^{\prime}\right), \\
& \quad=\Delta^{-3} \exp \left\{\frac{1}{\Delta} \sum_{i=1}^{2}\left[\mathbf{Q}_{i} \cdot \mathbf{Q}_{i}^{\prime}+\frac{1}{2}\left(\epsilon^{\prime} \mathbf{Q}_{i}^{2}+\epsilon \mathbf{Q}_{i}^{\prime 2}\right)\right]\right\},
\end{aligned}
$$

where

$$
\Delta=1-\epsilon \epsilon^{\prime} .
$$

After substitution of Eq. (42) in Eq. (48), one obtains

$$
\begin{aligned}
I\left(\epsilon, S, \mathbf{Q}_{1}, \mathbf{Q}_{2} ; \epsilon^{\prime}, S^{\prime}, \mathbf{Q}_{1}^{\prime}, \mathbf{Q}_{2}^{\prime}\right) \\
\quad=\Delta^{-3} \exp \left\{\frac{S S^{\prime}}{\Delta} \check{\mathbf{Q}}_{1} \cdot \check{\mathbf{Q}}_{1}^{\prime}\right\} \exp \left\{\frac{S S^{\prime}}{\Delta} \check{\mathbf{Q}}_{2} \cdot \check{\mathbf{Q}}_{2}^{\prime}\right\} .
\end{aligned}
$$

It is interesting to note that the arguments of the exponential factors are diagonal in the generator coordinates $S$ and $S^{\prime}$. To obtain an expansion in terms of angular momenta, the well-known relation

$$
\exp \{\mathbf{a} \cdot \mathbf{b}\}=4 \pi \sum_{l m} i_{l}(a b) Y_{l m}^{*}(\hat{\mathbf{a}}) Y_{l m}(\hat{\mathbf{b}})
$$

can be applied, where $i_{l}(x)$ is the modified spherical bessel function of the first kind. Substitution of Eq. (50) in Eq. (49), and applying traditional angular momentum coupling techniques leads to

$$
\begin{aligned}
& I\left(\epsilon, S, \mathbf{Q}_{1}, \mathbf{Q}_{2} ; \epsilon^{\prime}, S^{\prime}, \mathbf{Q}_{1}^{\prime}, \mathbf{Q}_{2}^{\prime}\right) \\
&=(4 \pi)^{2} \sum_{l_{1}, m_{1}, l_{2}, m_{2}} i_{l_{1}}\left(\frac{S S^{\prime}}{\Delta}\right) i_{l_{2}}\left(\frac{S S^{\prime}}{\Delta}\right) Y_{l_{1} m_{1}}^{*}\left(\check{\mathbf{Q}}_{1}\right) \\
& \times Y_{l_{2} m_{2}}^{*}\left(\check{\mathbf{Q}}_{2}\right) Y_{l_{1} m_{1}}\left(\check{\mathbf{Q}}_{1}^{\prime}\right) Y_{l_{2} m_{2}}\left(\check{\mathbf{Q}}_{2}^{\prime}\right) \\
&=(4 \pi)^{2} \sum_{\left(l_{1} l_{2}\right) L M} i_{l_{1}}\left(\frac{S S^{\prime}}{\Delta}\right) i_{l_{2}}\left(\frac{S S^{\prime}}{\Delta}\right) \\
& \times\left\{Y_{l_{1}}^{*}\left(\check{\mathbf{Q}}_{1}\right) \times Y_{l_{2}}^{*}\left(\check{\mathbf{Q}}_{2}\right)\right\}_{L M}\left\{Y_{l_{1}}\left(\check{\mathbf{Q}}_{1}^{\prime}\right) Y_{l_{2}}\left(\check{\mathbf{Q}}_{2}^{\prime}\right)\right\}_{L M} .
\end{aligned}
$$

The reduced generating function [cf. Eq. (47)] then becomes

$\mathcal{I}_{\left(l_{1} l_{2}\right) L M ;\left(l_{1} l_{2}\right) L M}\left(\epsilon, S ; \epsilon^{\prime}, S^{\prime}\right)=(4 \pi)^{2} \Delta^{-3} i_{l_{1}}\left(\frac{S S^{\prime}}{\Delta}\right) i_{l_{2}}\left(\frac{S S^{\prime}}{\Delta}\right)$.

This reduced generating function (52) is diagonal in the partial angular momenta $l_{1}$ and $l_{2}$, and independent of total angular momentum $L$, thus valid for all angular momenta $L$ compatible with $l_{1}$ and $l_{2}$.

The matrix elements with quantum numbers $K$ (also $n$ ) can now be obtained through a standard procedure, e.g., by differentiating Eq. (52) with respect to $S$ and $S^{\prime}$ ( $\epsilon$ and $\epsilon^{\prime}$ for $n)$. In particular, as Eq. (52) depends on the product of $S$ and $S^{\prime}$ only, the overlap is diagonal in $K$. Likewise, the dependence on $\epsilon$ and $\epsilon^{\prime}$ appears as a product in the factor $\Delta$, so that the overlap is again diagonal in $n$. The fact that the generated overlap matrix elements are diagonal is a confirmation of the fact that these matrix elements were generated in an orthogonal basis. The calculation of the matrix is however not unimportant, as it provides a straightforward way to obtain the norm of the generator coordinate basis function (44). 


\section{B. Matrix elements for the kinetic energy}

In order to calculate the kinetic energy one can use the properties of the oscillator basis. The matrix elements of the kinetic energy of relative motion of the clusters are related to those of the oscillator potential by the virial theorem

$$
\begin{gathered}
\left\langle N\left|\hat{T}_{R}\right| N\right\rangle=\left\langle N\left|\hat{V}_{O}\right| N\right\rangle=\frac{1}{2} E_{N} \\
\left\langle N \pm 2\left|\hat{T}_{R}\right| N\right\rangle=-\left\langle N \pm 2\left|\hat{V}_{O}\right| N\right\rangle,
\end{gathered}
$$

where

$$
\hat{V}_{O}=\frac{1}{2} \hbar \omega \sum_{i=1} \mathbf{q}_{i}^{2}
$$

and $E_{N}=\hbar \omega[N+3]=\hbar \omega[2 n+K+3]$ is the oscillator energy of relative motion.

One easily obtains a representation of the oscillator potential in the manifold spanned by the generating function (43):

$$
\begin{aligned}
\left(\mathbf{q}_{1}^{2}+\right. & \left.\mathbf{q}_{2}^{2}\right) \Psi\left(\mathbf{q}_{1}, \mathbf{q}_{2} \mid \epsilon, S ; \check{\mathbf{Q}}_{1}, \check{\mathbf{Q}}_{2}\right) \\
= & {\left[(1+\boldsymbol{\epsilon})^{2} \frac{d}{d \boldsymbol{\epsilon}}+(1+\boldsymbol{\epsilon}) S \frac{d}{d S}+3(1+\boldsymbol{\epsilon})\right] } \\
& \times \Psi\left(\mathbf{q}_{1}, \mathbf{q}_{2} \mid \epsilon, S ; \check{\mathbf{Q}}_{1}, \check{\mathbf{Q}}_{2}\right)
\end{aligned}
$$

so that the kinetic energy operator $\hat{T}_{R}$ can be represented by

$$
\mathcal{T}_{R}=\frac{1}{2} \hbar \omega\left[-(1-\epsilon)^{2} \frac{d}{d \epsilon}+(1-\epsilon) S \frac{d}{d S}+3(1-\epsilon)\right] .
$$

In a wider context, one can associate this operator with the generators $\mathcal{R}^{(+)}, \mathcal{R}^{(-)}$, and $\mathcal{R}^{(0)}$,

$$
\begin{gathered}
\mathcal{R}^{(+)}=\left[\epsilon^{2} \frac{d}{d \epsilon}+\epsilon S \frac{d}{d S}+3 \epsilon\right], \\
\mathcal{R}^{(-)}=\left[\frac{d}{d \epsilon}\right], \\
\mathcal{R}^{(0)}=\left[2 \epsilon \frac{d}{d \epsilon}+S \frac{d}{d S}+3\right],
\end{gathered}
$$

of the $\operatorname{Sp}(2, R)$ group classifying the space of relative motion of three-particle states, and whose irreducible representations are labeled by hypermomentum $K$ so that

$$
\begin{aligned}
& \mathcal{V}_{R}=\frac{1}{2} \hbar \omega\left[\mathcal{R}^{(0)}+\mathcal{R}^{(+)}+\mathcal{R}^{(-)}\right], \\
& \mathcal{T}_{R}=\frac{1}{2} \hbar \omega\left[\mathcal{R}^{(0)}-\mathcal{R}^{(+)}-\mathcal{R}^{(-)}\right] .
\end{aligned}
$$

Using again the shorthand notation $\nu_{0}=\left(l_{1} l_{2}\right) L M$, matrix elements are then readily found to be

$$
\begin{gathered}
\left\langle n, K, \nu_{0}\left|\hat{T}_{R}\right| n, K, \nu_{0}\right\rangle=\frac{1}{2} \hbar \omega[2 n+K+3], \\
\left\langle n+1, K, \nu_{0}\left|\hat{T}_{R}\right| n, K, \nu_{0}\right\rangle=-\frac{1}{2} \hbar \omega \sqrt{(n+1)(n+K+3),} \\
\left\langle n-1, K, \nu_{0}\left|\hat{T}_{R}\right| n, K, \nu_{0}\right\rangle=-\frac{1}{2} \hbar \omega \sqrt{n(n+K+2)} .
\end{gathered}
$$

The full kinetic energy of the three cluster system must include the internal kinetic energy of the clusters: $\hat{T}=\hat{T}_{R}$ $+\hat{T}_{c l}$. As we consider frozen $s$ clusters only, this contribution is purely diagonal and equal to

$$
T_{c l}=\frac{3}{4} \hbar \omega \sum_{i=1}^{3}\left(A_{i}-1\right)=\frac{3}{4} \hbar \omega(A-3) .
$$

\section{Matrix elements for a Gaussian potential}

For a Gaussian two-body interaction with strength $V_{0}$ and range $a$,

$$
V\left(\mathbf{r}_{i}, \mathbf{r}_{j}\right)=V_{0} \exp \left\{-\frac{\left(\mathbf{r}_{i}-\mathbf{r}_{j}\right)^{2}}{a^{2}}\right\}
$$

the folding potential (5) for $(0 s)$ clusters can be calculated analytically. The result obtained from Eq. (6) is again of a Gaussian form but with a modified strength and interaction length now also depending on the oscillator parameter $b$

$$
\begin{gathered}
V^{(F)}\left(\mathbf{R}_{\tau v}\right)=V_{0} z^{3 / 2} \exp \left\{-\frac{z}{a^{2}} \mathbf{R}_{\tau v}^{2}\right\} \\
z=\left(1+\frac{b^{2}}{a^{2}}\left[2-\mu_{\tau v}^{-1}\right]\right)^{-1}, \quad \mu_{\tau v}=\frac{A_{\tau} A_{v}}{A_{\tau}+A_{v}} .
\end{gathered}
$$

The matrix element of a Gaussian potential between two generating functions of the form (40) is not necessarily diagonal in terms of the chosen Jacobi coordinates. In the folding approximation one can, however, easily find a set Jacobi coordinates in which the potential matrix element is diagonal. The two sets of Jacobi coordinates will then be related by an orthogonal transformation.

In what follows, we distinguish the two types of Jacobi coordinates as follows: the original coordinates are denoted by $\mathbf{q}$ and were introduced in Sec. II A, and shown in Fig. 1; the diagonalizing coordinates will be denoted by $\mathbf{x}$. An explicit example of the latter are easily obtained. Indeed, for any choice of two clusters $i$ and $j(i \neq j), k$ being the third particle, one obtains a system of coordinates $\mathbf{x}$, uniquely defined by index $k$, as follows:

$$
\begin{gathered}
\mathbf{x}_{1}=\sqrt{c_{1, k}}\left(\mathbf{r}_{i}-\mathbf{r}_{j}\right), \\
\mathbf{x}_{2}=\sqrt{c_{2, k}}\left(\mathbf{r}_{k}-\frac{A_{i} \mathbf{r}_{i}+A_{j} \mathbf{r}_{j}}{A_{i}+A_{j}}\right),
\end{gathered}
$$




$$
c_{1, k}=\mu_{i j}=\frac{A_{i} A_{j}}{A_{i}+A_{j}}, \quad c_{2, k}=\frac{A_{k}\left(A_{i}+A_{j}\right)}{A_{i}+A_{j}+A_{k}} .
$$

Each such Jacobi coordinate system $k$ leads to a diagonal representation for the potential energy between clusters $i$ and $j$. As both Jacobi systems $\mathbf{q}$ and $\mathbf{x}$ are related through an orthogonal transformation, we can invoke the Raynal-Revai theorem [36]. The latter states that any orthogonal transformation of Jacobi coordinates leads to an orthogonal transformation of the hyperspherical harmonics (39) in the wave function (37), preserving the hypermomentum quantum number $K$,

$$
H_{K}^{\left(l_{1} l_{2}\right) L M}\left(\theta_{\mathbf{q}}, \check{\mathbf{q}}_{1}, \check{\mathbf{q}}_{2}\right)=\sum_{\lambda_{1} \lambda_{2}} O_{\lambda_{1} \lambda_{2}}^{l_{1} l_{2}} H_{K}^{\left(\lambda_{1} \lambda_{2}\right) L M}\left(\theta_{\mathbf{x}}, \check{\mathbf{x}}_{1}, \check{\mathbf{x}}_{2}\right) .
$$

The $O_{\lambda_{1} \lambda_{2}}^{l_{1} l_{2}}$ are known as the Raynal-Revai coefficients [36].

This transformation can then be used to obtain the matrix elements of the potential in the original set of coordinates $\mathbf{q}$ through

$$
\begin{aligned}
\langle n, K, & \left.\left(l_{1} l_{2}\right) L M|\hat{V}| n^{\prime}, K^{\prime},\left(l_{1}^{\prime} l_{2}^{\prime}\right) L M\right\rangle_{(\mathbf{q})} \\
= & \sum_{\lambda_{1} \lambda_{2}} \sum_{\lambda_{1}^{\prime} \lambda_{2}^{\prime}} O_{\lambda_{1} \lambda_{2}}^{l_{1} l_{2}} O_{\lambda_{1}^{\prime} \lambda_{2}^{\prime}}^{l_{1}^{\prime} l_{2}^{\prime}} \\
& \times\left\langle n, K,\left(\lambda_{1} \lambda_{2}\right) L M|\hat{V}| n^{\prime}, K^{\prime},\left(\lambda_{1}^{\prime} \lambda_{2}^{\prime}\right) L M\right\rangle_{(\mathbf{x})},
\end{aligned}
$$

where the matrix element on the right-hand side, which is diagonal in the $\mathbf{x}$ representation, can be calculated in a straightforward way.

To obtain the latter matrix element we consider a generating function of an identical structure as Eq. (40), in which we replace the $\mathbf{q}$ coordinates and $\mathbf{Q}$ generator coordinates by $\mathbf{X}$ and $\mathbf{X}$. A generating function for the two-body matrix elements is then easily obtained as a product of two integrals over $\mathbf{x}_{1}$ and $\mathbf{x}_{2}$ leading to

$$
\begin{gathered}
V\left(\epsilon, S, \check{\mathbf{X}}_{1}, \check{\mathbf{X}}_{2} ; \epsilon^{\prime}, S^{\prime}, \check{\mathbf{X}}_{1}^{\prime}, \check{\mathbf{X}}_{2}^{\prime}\right) \\
=V_{p r e} \exp \left\{\frac{S S^{\prime}}{\Lambda} \check{\mathbf{X}}_{1} \cdot \check{\mathbf{X}}_{1}^{\prime}\right\} \exp \left\{\frac{S S^{\prime}}{\Delta} \check{\mathbf{X}}_{2} \cdot \check{\mathbf{X}}_{2}^{\prime}\right\} \\
V_{p r e}=V_{0}(\Delta \Lambda)^{-3 / 2} \exp \left\{-\frac{\gamma}{2 \Delta \Lambda}\left[\left(\xi^{\prime} S\right)^{2}+\left(\xi S^{\prime}\right)^{2}\right]\right\}, \\
\xi=1+\epsilon, \quad \xi^{\prime}=1+\epsilon^{\prime}, \\
\Delta=1-\epsilon \epsilon^{\prime}=\xi+\xi^{\prime}-\xi \xi^{\prime}, \\
\Lambda=\Delta+\gamma \xi \xi^{\prime}, \\
\gamma=\frac{b^{2}}{a^{2}} \frac{1}{c_{1, \alpha}} .
\end{gathered}
$$

By using Eq. (50), one again eliminates factorized terms and sums to obtain the following reduced generating function [cf. (47)], in complete analogy to the procedure taken for Eq. (52):

$$
\mathcal{V}_{\left(l_{1} l_{2}\right) L M ;\left(l_{1} l_{2}\right) L M}\left(\epsilon, S ; \epsilon^{\prime}, S^{\prime}\right)=V_{p r e} i_{l_{1}}\left(\frac{S S^{\prime}}{\Lambda}\right) i_{l_{2}}\left(\frac{S S^{\prime}}{\Delta}\right) \text {. }
$$

As was the case with the overlap, the matrix elements of the potential in the $\mathbf{x}$ coordinate system are diagonal with respect to the partial angular momenta $l_{1}$ and $l_{2}$ and do not depend directly upon the total angular momentum $L$; this is a direct consequence of the characteristics of the operator. Expression (69) is again valid for all values of the total angular momenta $L$ that are compatible with the given partial angular momenta $l_{1}$ and $l_{2}$, and thus represents a generating function for matrix elements with specific total angular momentum $L$.

One obtains matrix elements with specific $K$ and $n$ quantum numbers through the standard procedures (differentiation, recurrence relations, ... . ). So, for example, one obtains

$$
\begin{aligned}
\langle n, & \left.,\left(l_{1} l_{2}\right) L M|\hat{V}| n^{\prime}, K^{\prime},\left(l_{1} l_{2}\right) L M\right\rangle_{(\mathbf{x})} \\
= & \left(\frac{d}{d \epsilon}\right)^{n}\left(\frac{d}{d \epsilon^{\prime}}\right)^{n^{\prime}}\left(\frac{d}{d S}\right)^{K}\left(\frac{d}{d S^{\prime}}\right)^{K^{\prime}} \\
& \times\left.\mathcal{V}_{\left(l_{1} l_{2}\right) L M ;\left(l_{1} l_{2}\right) L M}\left(\epsilon, S ; \epsilon^{\prime}, S^{\prime}\right)\right|_{\substack{S=S^{\prime}=0 \\
\epsilon=\epsilon^{\prime}=0}}
\end{aligned} .
$$

\section{Matrix elements for the Coulomb potential}

The Coulomb interaction in the folding approximation between two clusters with $Z_{\tau}$ and $Z_{v}$ number of protons is given by Eq. (6) as

$$
\begin{aligned}
V_{C}^{(F)}\left(\mathbf{R}_{\tau v}\right)= & Z_{\tau} Z_{v} e^{2} \sum_{i \in A_{\tau}} \sum_{j \in A_{v}} \int d \tau_{\tau} d \tau_{v}\left|\Psi_{\tau}\left(A_{\tau}\right)\right|^{2} \\
& \times \frac{1}{\left|\mathbf{r}_{i}-\mathbf{r}_{j}+\mathbf{R}_{\tau v}\right|}\left|\Psi_{v}\left(A_{v}\right)\right|^{2} .
\end{aligned}
$$

A straightforward calculation of its matrix elements is impractical and very tedious. By, however, using the following Gauss transform:

$$
\frac{1}{r}=\frac{2}{\sqrt{\pi}} \int_{0}^{\infty} d x \exp \left\{-r^{2} x^{2}\right\}
$$

one rewrites Eq. (72) as

$$
\begin{aligned}
V_{C}^{(F)}(\tau v)= & \frac{2 Z_{\tau} Z_{v} e^{2}}{\sqrt{\pi}} \sum_{i \in A_{\tau}} \sum_{j \in A_{v}} \int d \tau_{\tau} d \tau_{v}\left|\Psi_{\tau}\left(A_{\tau}\right)\right|^{2} \\
& \times \int_{0}^{\infty} d x \exp \left\{-\left(\mathbf{r}_{i}-\mathbf{r}_{j}+\mathbf{R}_{\tau v}\right)^{2} x^{2}\right\}\left|\Psi_{v}\left(A_{v}\right)\right|^{2} \\
= & \frac{2 Z_{\tau} Z_{v} e^{2}}{\sqrt{\pi} b} \int_{0}^{\infty} d \gamma z^{3 / 2} \exp \left\{-\frac{R_{\tau v}^{2}}{b^{2}} \gamma^{2} z\right\}
\end{aligned}
$$


where

$$
z=\left(1+t \gamma^{2}\right)^{-1}, \quad t=2-\mu_{\tau v}^{-1}, \quad \gamma=b x
$$

and its matrix elements can be obtained by integrating matrix elements (depending on $z$ ) of the Gaussian potential.

Introducing the integration variable $s$

$$
s=\frac{t \gamma^{2}}{1+t \gamma^{2}},
$$

transforms Eq. (74) to

$$
\begin{aligned}
V_{C}^{(F)}(\tau v) & =\frac{2 Z_{\tau} Z_{v} e^{2}}{\sqrt{\pi} b} \frac{1}{2 \sqrt{t}} \int_{0}^{1} d s s^{-1 / 2} \exp \left\{-\frac{R_{\tau v}^{2}}{t b^{2}} s\right\} \\
& =\frac{Z_{\tau} Z_{v} e^{2}}{R_{\tau v}} \operatorname{erf}\left(\sqrt{\frac{R_{\tau v}^{2}}{t b^{2}}}\right) .
\end{aligned}
$$

This form shows that the integration can be reduced to a finite interval. It also shows that the Coulomb interaction between clusters does not behave as $R_{\tau v}^{-1}$ as could be expected. For very large value of $R_{\tau v}$, however, Eq. (77) properly reduces to $Z_{\tau} Z_{v} e^{2} / R_{\tau v}$, due to the asymptotic form of the error function.

\section{E. Asymptotic behavior of the potential contributions}

As the folding model is used for defining the asymptotic channels, it is clear that the asymptotic behavior of the potential energy matrix elements in this model will be of vital importance for the rate of convergence of the AM solutions. The effective potential in terms of the hyper-radius $\rho$ is defined by integrating the folding potential over all hyperangles as formally indicated by

$$
\begin{aligned}
W(\rho)=\sum_{\tau, v} W_{\tau v}(\rho) & \equiv W_{K, l_{1}, l_{2}}(\rho) \\
& =\left\langle K, l_{1}, l_{2}\left|\sum_{\tau, v} \hat{V}\left(\mathbf{R}_{\tau v}\right)\right| K, l_{1}, l_{2}\right\rangle
\end{aligned}
$$

Its asymptotic behavior is then obtained for large values of $\rho$.

In the diagonal representation with Jacobi coordinates $\mathbf{x}$ the calculation of $W(\rho)$ between one pair of clusters $\tau$ and $v$ amounts to a straightforward integration over the hyperangles.

The Gaussian interaction then exhibits the following asymptotic behavior:

$$
\begin{aligned}
W_{\tau v}(\rho) \approx & (-1)^{K-l_{1}-l_{2}} V_{0} N_{K}^{l_{1} l_{2}} N_{K}^{l_{1} l_{2}}\left(\frac{1}{2}\right)^{K+3} \Gamma\left(l_{1}+\frac{3}{2}\right) \\
& \times\left(\frac{\sqrt{\mu_{\tau v}} a}{\rho}\right)^{2 l_{1}+3}\left(\frac{K+l_{1}-l_{2}+1}{2}\right) \\
& \times\left(\begin{array}{c}
\frac{K+l_{1}-l_{2}+1}{2} \\
K-l_{1}-l_{2}
\end{array}\right),
\end{aligned}
$$

which indeed shows a worst-case behavior (for $l_{1}=0$ ) of the form $1 / \rho^{3}$ as predicted in Sec. III D.

The analogous evaluation for the Coulomb interaction leads to the following (exact) expression:

$$
W_{\tau v}(\rho)=\frac{Z_{K, l_{1}, l_{2}}^{e f f}}{\rho}
$$

with

$$
\begin{aligned}
Z_{K, l_{1}, l_{2}}^{e f f}= & \frac{1}{2} Z_{\tau} Z_{v} e^{2} \sqrt{\mu_{\tau v}} \\
& \times \sum_{n, m=0}^{\left(K-l_{1}-l_{2}\right) / 2}(-)^{K-l_{1}-l_{2}-n-m}\left(\frac{K-l_{1}+l_{2}+1}{2}\right) \\
& \times\left(\frac{K-l_{1}+l_{2}+1}{2}\right)\left(\frac{K+l_{1}-l_{2}+1}{2}\right)\left(\frac{K-l_{1}-l_{2}}{2}-n\right) \\
& \times\left(\frac{K+l_{1}-l_{2}+1}{2}\right) \\
& B\left(K-l_{1}-n-m+\frac{3}{2}, n+m+l_{1}+1\right)
\end{aligned}
$$

and $B$ stands for the beta function.

These results corroborate the fact that special care should be taken to get properly convergent results, even more critically when a Coulomb contribution between the clusters is present.

\section{MATRIX ELEMENTS WITH FULL ANTISYMMETRIZATION}

When considering full antisymmetrization between all particles of the three-cluster wave function the normalization of the basis states becomes a nontrivial problem. The overlap, i.e., the matrix representation of the antisymmetrization operator $\mathcal{A}$, needs to be explicitly calculated.

As was carried out in the previous section one would normally start from the generating state (40) to obtain gen- 
erating matrix elements in terms of $\epsilon$ and $\epsilon^{\prime}$, facilitating the treatment of the hyperangular coordinates. We propose an alternative representation for the scaled generating state (40) which is more suited to our calculations, and hereto introduce the following integral transformation:

$$
\Psi(\mathbf{q} \mid \epsilon, \mathbf{Q})=\int d \mathbf{k} \exp \left\{-k^{2}\right\} \Psi(\mathbf{q} ; \mathbf{Q}+\sqrt{2 \epsilon} \mathbf{k}) .
$$

This allows us to obtain Eq. (40) by scaling on the generator coordinate only. In other words, generating matrix elements can be obtained with the simpler generating state (29) and scaled later on, reducing effectively the calculational burden.

\section{A. Matrix elements for the overlap}

Because the individual cluster states are Slater determinants, apart from the center-of-mass factor, one can use the familiar determinantal formulas to calculate the generating function. Starting then from the generating state (29) for the single-particle orbitals one obtains

$$
I\left(\mathbf{Q}_{1}, \mathbf{Q}_{2} ; \mathbf{Q}_{1}^{\prime}, \mathbf{Q}_{2}^{\prime}\right)=\sum_{\nu} D^{(\nu)} \exp \left\{\sum_{i, j=1}^{2} B_{i j}^{(\nu)} \mathbf{Q}_{i} \cdot \mathbf{Q}_{j}^{\prime}\right\} .
$$

The coefficients $D^{(\nu)}$ and $B_{i j}^{(\nu)}$ as well as the number of the terms depend on the specific type of three-cluster configuration, viz. the number of nucleons per cluster and their spinisospin quantum numbers.

In order to reduce Eq. (83) with respect to the angular quantum numbers it is profitable to diagonalize the forms in the exponentials of Eq. (83). This can easily be achieved by diagonalizing its 2 by 2 coefficient matrix $B_{i j}^{(\nu)}$. This again amounts to making an orthogonal transformation from the original Jacobi coordinates $\mathbf{q}$ to new Jacobi coordinates $\mathbf{x}$. This orthogonal transformation will induce a corresponding transformation of the basis functions which can be handled by the Raynal-Revai theorem as discussed in the previous section. The block-diagonal form of an exponential term can be written as

$$
\exp \left\{\sum_{i=1}^{2} \lambda_{i} \mathbf{X}_{i} \cdot \mathbf{X}_{i}^{\prime}\right\}
$$

We now introduce the scaling on $\epsilon$ and $\epsilon^{\prime}$ by carrying out the transformation (82) on both generator coordinates $\mathbf{X}$ and $\mathbf{X}^{\prime}$ for every (block-diagonal) term in Eq. (83), leading to

$$
\begin{aligned}
& I\left(\epsilon, \mathbf{X}_{1}, \mathbf{X}_{2} ; \epsilon^{\prime}, \mathbf{X}_{1}^{\prime}, \mathbf{X}_{2}^{\prime}\right) \\
& \quad=\left(\Delta_{1} \Delta_{2}\right)^{-3 / 2} \exp \left\{\sum_{i=1}^{2} \frac{\lambda_{i}}{\Delta_{i}}\left[\mathbf{X}_{i} \cdot \mathbf{X}_{i}^{\prime}+\frac{\lambda_{i}}{2}\left(\epsilon^{\prime} \mathbf{X}_{i}^{2}+\epsilon \mathbf{X}_{i}^{\prime 2}\right)\right]\right\},
\end{aligned}
$$

where

$$
\Delta_{i}=1-\lambda_{i}^{2} \epsilon \epsilon^{\prime}
$$

In order to further reduce the generating function in the partial angular momenta $l_{1}$ and $l_{2}$ we substitute Eq. (42) in Eq. $(85)$,

$$
\begin{aligned}
& I\left(\epsilon, S, \check{\mathbf{X}}_{1}, \check{\mathbf{X}}_{2} ; \epsilon^{\prime}, S^{\prime}, \check{\mathbf{X}}_{1}^{\prime}, \check{\mathbf{X}}_{2}^{\prime}\right) \\
&=\left(\Delta_{1} \Delta_{2}\right)^{-3 / 2} \exp \left\{\frac{\lambda_{1}^{2}-\lambda_{2}^{2}}{2 \Delta_{1} \Delta_{2}}\left[S^{2} \epsilon^{\prime}+S^{\prime} \check{\epsilon}^{\prime}\right]\right\} \\
& \times \exp \left\{\frac{\lambda_{1}}{\Delta_{1}} S S^{\prime} \check{\mathbf{X}}_{1} \cdot \check{\mathbf{X}}_{1}^{\prime}\right\} \exp \left\{\frac{\lambda_{2}}{\Delta_{2}} S S^{\prime} \check{\mathbf{X}}_{2} \cdot \check{\mathbf{X}}_{2}^{\prime}\right\} .
\end{aligned}
$$

By using Eq. (50) this produces in complete analogy to Eq. (52) a generating function for all total angular momenta $L$ compatible with $l_{1}$ and $l_{2}$ :

$$
\begin{aligned}
\mathcal{I}_{\left(l_{1}, l_{2}\right) L M ;\left(l_{1} l_{2}\right) L M}\left(\epsilon, S ; \epsilon^{\prime}, S^{\prime}\right) & \\
= & (4 \pi)^{2}\left(\Delta_{1} \Delta_{2}\right)^{-3 / 2} \exp \left\{\frac{\lambda_{1}^{2}-\lambda_{2}^{2}}{2 \Delta_{1} \Delta_{2}}\left[S^{2} \epsilon^{\prime}+S^{\prime} \epsilon^{2} \epsilon\right]\right\} \\
& \times i_{l_{1}}\left(\frac{\lambda_{1}}{\Delta_{1}} S S^{\prime}\right) i_{l_{2}}\left(\frac{\lambda_{2}}{\Delta_{2}} S S^{\prime}\right)
\end{aligned}
$$

If $\lambda_{1}=\lambda_{2}$, the overlap depends on the factor $S S^{\prime}$ only and is therefore diagonal with respect to hypermomentum $K$, as was the case in the folding approximation. In the general case one will have $\lambda_{1} \neq \lambda_{2}$, and Eq. (87) generates nondiagonal matrix elements in $K$. Hypermomentum is thus no longer a good quantum number for three-cluster systems, contrary to the folding approximation.

The generating function for the overlap with $K=0$, and consequently for angular momenta $l_{1}=l_{2}=L=0$, is obtained immediately by putting $S=S^{\prime}=0$. This simplicity is an indication of the very suitable form of our generating function.

The matrix elements $I_{n, n^{\prime}}^{K, \nu_{0} ; K^{\prime}, \nu_{0}}$ can now again be generated by a standard procedure, such as differentiation or recurrence relations.

\section{B. Matrix elements for the kinetic energy}

The matrix elements of the kinetic energy operator can be derived without use of a generating function. One of the effects of the antisymmetrization operator is to mix basis states within a fixed oscillator shell. The "diagonal" (i.e., within a shell) and "off-diagonal" (within neighboring shells) kinetic energy matrix elements are easily found to be connected to the matrix elements of the overlap by

$$
\begin{aligned}
T_{n, n^{\prime}}^{K, \nu_{0} ; K^{\prime}, \nu_{0}^{\prime}}= & \frac{1}{2} \hbar \omega\left[2 n+K+3+\frac{3}{2}(A-3)\right] \\
& \times I_{n, n^{\prime}}^{K, \nu_{0} ; K^{\prime}, \nu_{0}^{\prime}} \delta_{2 n+K, 2 n^{\prime}+K^{\prime}}, \\
T_{n+1, n^{\prime}}^{K, \nu_{0} ; K^{\prime}, \nu_{0}^{\prime}}= & -\frac{1}{2} \hbar \omega \sqrt{(n+1)(n+K+3)} \\
& \times I_{n, n^{\prime}}^{K, \nu_{0} ; K^{\prime}, \nu_{0}^{\prime}} \delta_{2 n+K, 2 n^{\prime}+K^{\prime}},
\end{aligned}
$$


where the restriction on the quantum numbers to remain on the same oscillator shell $\left(2 n+K=2 n^{\prime}+K^{\prime}\right)$ has been accounted for.

\section{Matrix elements for a Gaussian potential}

If one considers a Gaussian form for the nucleon-nucleon potential, and calculates a generating matrix element for the interaction using Slater determinants with individual orbitals of the form (29) one obtains terms of the form

$$
\begin{aligned}
V\left(\mathbf{Q}_{1}, \mathbf{Q}_{2} ; \mathbf{Q}_{1}^{\prime}, \mathbf{Q}_{2}^{\prime}\right) & \\
= & V_{0}(1-\zeta)^{3 / 2} \exp \left\{-\zeta\left[\sum_{i=1}^{2}\left(C_{i} \mathbf{Q}_{i}+C_{i}^{\prime} \mathbf{Q}_{i}^{\prime}\right)\right]^{2}\right. \\
& \left.+\sum_{i, j=1}^{2} B_{i j} \mathbf{Q}_{i} \cdot \mathbf{Q}_{j}^{\prime}\right\}
\end{aligned}
$$

where

$$
\zeta=\frac{2 b^{2}}{2 b^{2}+a^{2}}
$$

and $V_{0}$ stands for any of the even $\left(V_{13}\right.$ and $\left.V_{31}\right)$ and odd ( $V_{11}$ and $V_{33}$ ) components of the $N N$ interaction. Again $b$ is the oscillator radius and $a$ the range of the potential well. The first term in the exponent of Eq. (88) contains the factor $\zeta$ and the vectors $C_{1} \mathbf{Q}_{1}+C_{2} \mathbf{Q}_{2}$ and $C_{1}^{\prime} \mathbf{Q}_{1}^{\prime}+C_{2}^{\prime} \mathbf{Q}_{2}^{\prime}$. The latter have a simple meaning: they define the distance between the two clusters which respectively contain one of the nucleons in the interacting pair.

In the diagonal representation (88) becomes

$$
\begin{aligned}
V\left(\mathbf{X}_{1}, \mathbf{X}_{2} ; \mathbf{X}_{1}^{\prime}, \mathbf{X}_{2}^{\prime}\right)= & V_{0} \exp \left\{-\zeta\left[\sum_{i=1}^{2}\left(G_{i} \mathbf{X}_{i}+G_{i}^{\prime} \mathbf{X}_{i}^{\prime}\right)\right]^{2}\right. \\
& \left.+\sum_{i=1}^{2} \lambda_{i} \mathbf{X}_{i} \cdot \mathbf{X}_{i}^{\prime}\right)
\end{aligned}
$$

and the coefficients $C_{i}, C_{i}^{\prime}$ and $G_{i}, G_{i}^{\prime}$ are trivially related by the orthogonal diagonalizing transformation.

In order to eliminate crossterms in $\mathbf{X}_{1} \cdot \mathbf{X}_{2}$ and $\mathbf{X}_{1}^{\prime} \cdot \mathbf{X}_{2}^{\prime}$ we introduce an additional transformation through the integral identity

$$
e^{-\mathbf{a}^{2}}=\frac{1}{\pi^{3 / 2}} \int_{-\infty}^{\infty} d \mathbf{Z} \exp \left\{-Z^{2}+2 i(\mathbf{Z} \cdot \mathbf{a})\right\}
$$

leading to the following integral form for the block (90):

$$
\begin{aligned}
V\left(\mathbf{X}_{1}, \mathbf{X}_{2} ; \mathbf{X}_{1}^{\prime}, \mathbf{X}_{2}^{\prime}\right) \\
=V_{0} \frac{1}{\pi^{3 / 2}} \exp \left\{\sum_{i=1}^{2} \lambda_{i} \mathbf{X}_{i} \cdot \mathbf{X}_{i}^{\prime}\right\} \int_{-\infty}^{\infty} d \mathbf{Z} \\
\quad \times \exp \left\{-Z^{2}+2 i \zeta \sum_{i=1}^{2}\left(G_{i} \mathbf{Z} \cdot \mathbf{X}_{i}+G_{i}^{\prime} \mathbf{Z} \cdot \mathbf{X}_{i}^{\prime}\right)\right\} .
\end{aligned}
$$

Using Eq. (82) on both $\mathbf{X}_{i}$ and $\mathbf{X}_{i}^{\prime}$, one obtains after integrating over the corresponding $\mathbf{k}_{i}$ and $\mathbf{k}_{i}^{\prime}$

$$
\begin{aligned}
V\left(\epsilon ; \mathbf{X}_{1}, \mathbf{X} ; \epsilon^{\prime} ; \mathbf{X}_{1}^{\prime}, \mathbf{X}_{2}^{\prime}\right) \\
=\left(\Delta_{1} \Delta_{2}\right)^{-3 / 2} \exp \left\{\sum _ { i = 1 } ^ { 2 } \frac { \lambda _ { i } } { 2 \Delta _ { i } } \left(\epsilon^{\prime} \lambda_{i} \mathbf{X}_{i}^{2}+\epsilon \lambda_{i} \mathbf{X}_{i}^{\prime 2}\right.\right. \\
\left.\left.\quad+2 \mathbf{X}_{i} \cdot \mathbf{X}_{i}^{\prime}\right)\right\} \frac{1}{\pi^{3 / 2}} \int_{-\infty}^{\infty} d \mathbf{Z} \\
\quad \times \exp \left\{-\Delta \mathbf{Z}^{2}+2 i \zeta \sum_{i=1}^{2}\left(\xi_{i} \mathbf{Z} \cdot \mathbf{X}_{i}+\xi_{i}^{\prime} \mathbf{Z} \cdot \mathbf{X}_{i}^{\prime}\right)\right\},
\end{aligned}
$$

where again $\Delta_{i}=1-\lambda_{i}^{2} \epsilon \epsilon^{\prime}$ and

$$
\begin{gathered}
\xi_{i}=\frac{G_{i}+\epsilon^{\prime} G_{i}^{\prime} \lambda_{i}}{\Delta_{i}}, \quad \xi_{i}^{\prime}=\frac{G_{i}^{\prime}+\epsilon G_{i} \lambda_{i}}{\Delta_{i}}, \\
\Delta=\left\{1+\sum_{i=1}^{2} \frac{1}{\Delta_{i}}\left[\epsilon G_{i}^{2}+\epsilon^{\prime} G_{i}^{\prime 2}+2 \epsilon \epsilon^{\prime} \lambda_{i} G_{i} G_{i}^{\prime}\right]\right\} .
\end{gathered}
$$

To obtain a reduced generating function for specific angular momentum quantum numbers, we again use expansion formulas of an exponential in terms of spherical harmonics. For exponential terms with a real scalar product we use Eq. (50), whereas for exponential terms with an imaginary scalar product we use [37]

$$
\exp \{i \mathbf{a} \cdot \mathbf{b}\}=4 \pi \sum_{l m} i^{l} j_{l}(a b) Y_{l m}^{*}(\check{\mathbf{a}}) Y_{l m}(\check{\mathbf{b}})
$$

where $j_{l}(a b)$ is the well-known spherical Bessel function.

Applying both Eqs. (50) and (94) to Eq. (93) leads to

$V\left(\epsilon, \mathbf{X}_{1}, \mathbf{X} ; \epsilon^{\prime}, \mathbf{X}_{1}^{\prime}, \mathbf{X}_{2}^{\prime}\right)$

$$
\begin{aligned}
& =(4 \pi)^{6}\left(\Delta_{1} \Delta_{2}\right)^{-3 / 2} \exp \sum_{i=1}^{2}\left\{\frac{\lambda_{i}^{2}}{2 \Delta_{i}}\left(\epsilon^{\prime} \mathbf{X}_{i}^{2}+\epsilon \mathbf{X}_{i}^{\prime 2}\right)\right\}
\end{aligned}
$$

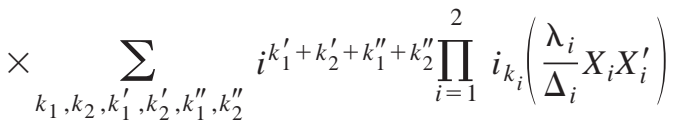

$$
\begin{aligned}
& \times \frac{1}{\pi^{3 / 2}} \int_{-\infty}^{\infty} d \mathbf{Z} \exp \left\{-\Delta Z^{2}\right\} P_{k_{1}, k_{2}, k_{1}^{\prime}, k_{2}^{\prime}, k_{1}^{\prime \prime}, k_{2}^{\prime \prime}} \\
& \times \prod_{i=1}^{2} j_{k_{i}^{\prime}}\left(2 \xi_{i} X_{i} Z\right) j_{k_{i}^{\prime \prime}}\left(2 \xi_{i}^{\prime} X_{i}^{\prime} Z\right) .
\end{aligned}
$$

Before performing the integration over $\mathbf{Z}$, we again make the substitution (42) in Eq. (95) to obtain 


$$
\begin{aligned}
V\left(\epsilon, S, \check{\mathbf{X}}_{1}, \check{\mathbf{X}}_{i} \epsilon^{\prime}, S^{\prime}, \check{\mathbf{X}}_{1}^{\prime}, \check{\mathbf{X}}_{2}^{\prime}\right)= & (4 \pi)^{6}\left(\Delta_{1} \Delta_{2}\right)^{-3 / 2} \exp \left\{\frac{\lambda_{1}^{2}-\lambda_{2}^{2}}{2 \Delta_{1} \Delta_{2}}\left(\epsilon^{\prime} S^{2}+\epsilon S^{\prime}\right\}\right\} \sum_{k_{1}, k_{2}, k_{1}^{\prime}, k_{2}^{\prime}, k_{1}^{\prime \prime}, k_{2}^{\prime \prime}} i^{k_{1}^{\prime}+k_{2}^{\prime}+k_{1}^{\prime \prime}+k_{2}^{\prime \prime}} \\
& \times i_{k_{1}}\left(\frac{\lambda_{1}}{\Delta_{1}} S S^{\prime}\right) i_{k_{2}}\left(\frac{\lambda_{2}}{\Delta_{2}} S S^{\prime}\right) \frac{1}{\pi^{3 / 2}} \int_{-\infty}^{\infty} d \mathbf{Z} \exp \left\{-\Delta Z^{2}\right\} j_{k_{1}^{\prime}}\left(2 \xi_{1} S Z\right) j_{k_{1}^{\prime \prime}}\left(2 \xi_{1}^{\prime} S^{\prime} Z\right) \\
& \times j_{k_{2}^{\prime}}\left(-i 2 \xi_{2} S Z\right) j_{k_{2}^{\prime \prime}}\left(i 2 \xi_{2}^{\prime} S^{\prime} Z\right) P_{k_{1}, k_{2}, k_{1}^{\prime}, k_{2}^{\prime}, k_{1}^{\prime \prime}, k_{2}^{\prime \prime},}
\end{aligned}
$$

where

$$
\begin{aligned}
P_{k_{1}, k_{2}, k_{1}^{\prime}, k_{2}^{\prime}, k_{1}^{\prime \prime}, k_{2}^{\prime \prime}}= & \prod_{i=1}^{2}\left(Y_{k_{i}}\left(\check{\mathbf{X}}_{i}\right) \cdot Y_{k_{i}}\left(\check{\mathbf{X}}_{i}^{\prime}\right)\right)\left(Y_{k_{i}^{\prime}}\left(\check{\mathbf{X}}_{i}\right) \cdot Y_{k_{i}^{\prime}}(\check{\mathbf{Z}})\right)\left(Y_{k_{i}^{\prime \prime}}(\check{\mathbf{Z}}) \cdot Y_{k_{i}^{\prime \prime}}\left(\check{\mathbf{X}}_{i}^{\prime}\right)\right) \\
= & \sum_{\left(l_{1}, l_{2}\right) L ;\left(l_{1}^{\prime}, l_{2}^{\prime}\right) L^{\prime} ; L^{\prime \prime}} \mathcal{P}_{\left\{k_{1}, k_{2}, k_{1}^{\prime}, k_{2}^{\prime}, k_{1}^{\prime \prime}, k_{2}^{\prime \prime}\right\} ;\left\{\left(l_{1}, l_{2}\right) L ;\left(l_{1}^{\prime}, l_{2}^{\prime}\right) L^{\prime} ; L^{\prime \prime}\right\}} \\
& \times\left(\left\{Y_{l_{1}}\left(\check{\mathbf{X}}_{1}\right) \times Y_{l_{2}}\left(\check{\mathbf{X}}_{2}\right)\right\}_{L} \cdot\left\{\left\{Y_{l_{1}^{\prime}}\left(\check{\mathbf{X}}_{1}^{\prime}\right) \times Y_{l_{2}^{\prime}}\left(\check{\mathbf{X}}_{2}^{\prime}\right)\right\}_{L^{\prime}} Y_{L^{\prime \prime}}(\check{\mathbf{Z}})\right\}_{L}\right) .
\end{aligned}
$$

The intermediate (but redundant) index $L^{\prime \prime}$ is connected to the integration variable $\mathbf{Z}$ of the integral transformation (91). Because of the orthogonality between spherical harmonics and of the scalar character of the potential operator $L$ and $L^{\prime}$ will be equal, and $L^{\prime \prime}=0$ after integration over $\mathbf{Z}$ : we therefore anticipate by simplifying Eq. (97) to

$$
\begin{aligned}
& P_{k_{1}, k_{2}, k_{1}^{\prime}, k_{2}^{\prime}, k_{1}^{\prime \prime}, k_{2}^{\prime \prime}} \\
& =\sum_{\left(l_{1}, l_{2}\right) L ;\left(l_{1}^{\prime}, l_{2}^{\prime}\right) L} \mathcal{P}_{\left\{k_{1}, k_{2}, k_{1}^{\prime}, k_{2}^{\prime}, k_{1}^{\prime \prime}, k_{2}^{\prime \prime}\right\} ;\left\{\left(l_{1}, l_{2}\right) L ;\left(l_{1}^{\prime}, l_{2}^{\prime}\right) L ; 0\right\}} \\
& \quad \times \frac{1}{\sqrt{4 \pi}}\left(\left\{Y_{l_{1}}\left(\check{\mathbf{X}}_{1}\right) \times Y_{l_{2}}\left(\check{\mathbf{X}}_{2}\right)\right\}_{L}\right. \\
& \left.\quad \times\left\{Y_{l_{1}^{\prime}}\left(\check{\mathbf{X}}_{1}^{\prime}\right) \times Y_{l_{2}^{\prime}}\left(\check{\mathbf{X}}_{2}^{\prime}\right)\right\}_{L}\right) .
\end{aligned}
$$

The expansion coefficients are then easily shown to be

$$
\begin{aligned}
\mathcal{P}_{\left\{k_{1}, k_{2}, k_{1}^{\prime}, k_{2}^{\prime}, k_{1}^{\prime \prime}, k_{2}^{\prime \prime}\right\} ;\left\{\left(l_{1}, l_{2}\right) L ;\left(l_{1}^{\prime}, l_{2}^{\prime}\right) L ; 0\right\}} & \sum_{k}(-1)^{l_{1}+l_{1}^{\prime}+k+L}\left\{\begin{array}{lll}
l_{2}^{\prime} & l_{2} & k \\
l_{1} & l_{1}^{\prime} & L
\end{array}\right\} C_{k 0 k 0}^{00} \\
& \times \prod_{i=1}^{2}(-1)^{k_{1}}\left(2 k_{i}+1\right)\left(2 k_{i}^{\prime}+1\right)\left(2 k_{i}^{\prime \prime}+1\right) \\
& \times\left\{\begin{array}{ccc}
k_{i}^{\prime \prime} & l_{i}^{\prime} & k_{i} \\
l_{i} & k_{i}^{\prime} & k
\end{array}\right\} C_{k_{i}^{\prime} 0 k_{i} 0}^{l_{i} 0} C_{k_{i}^{\prime \prime} 0 k_{i} 0}^{l_{i}^{\prime} 0} C_{k_{i}^{\prime} 0 k_{i}^{\prime \prime} 0}^{k 0} .
\end{aligned}
$$

Under the anticipative assumptions $L=L^{\prime}$ and $L^{\prime \prime}=0$, the integration over the angles $\check{\mathbf{Z}}$ in Eq. (96) is now trivial. The remaining integration over $Z$ is easily done after substituting the power expansions for the Bessel functions $j_{l}(x)$ and $i_{l}(x)$. The final result provides a tractable though very bulky result for $\mathcal{V}_{\left(l_{1} l_{2}\right) L M ;\left(l_{1} l_{2}\right) L M}\left(\epsilon, S ; \epsilon^{\prime}, S^{\prime}\right)$ that is not reproduced here, as it carries no further additional information.

Again, by using the standard procedures such as differentiation or recurrence relations one obtains the effective matrix elements. In particular, the form of Eq. (70) remains valid, though the analytic differentiation preferably should be performed within an algebraic package such as MATHEMATICA or MAPLE due to the bulkiness of the formulas.

\section{Matrix elements for the Coulomb potential}

The matrix elements of the Coulomb potential can now be most easily obtained from the Gaussian results. We consider again the Gaussian integral representation

$$
\frac{1}{\left|\mathbf{r}_{i}-\mathbf{r}_{j}\right|}=\frac{2}{\sqrt{\pi}} \int_{0}^{\infty} d x \exp \left\{-x^{2}\left(\mathbf{r}_{i}-\mathbf{r}_{j}\right)^{2}\right\}
$$

We use Eq. (96), replace $a^{2}$ by $1 / x^{2}$, and introduce a new integration variable $t=\gamma /(1-\gamma)$ where $\gamma=x^{2} b^{2}$, to obtain symbolically

$$
\begin{aligned}
& V_{C}\left(\epsilon, S, \check{\mathbf{X}}_{1}, \check{\mathbf{X}}_{2} ; \epsilon^{\prime}, S^{\prime}, \check{\mathbf{X}}_{1}^{\prime}, \check{\mathbf{X}}_{2}^{\prime}\right) \\
& \quad=\frac{2}{\sqrt{\pi}} \int_{0}^{\infty} d x V\left(\epsilon, S, \check{\mathbf{X}}_{1}, \check{\mathbf{X}}_{2} ; \epsilon^{\prime}, S^{\prime}, \check{\mathbf{X}}_{1}^{\prime}, \check{\mathbf{X}}_{2}^{\prime}\right) \\
& \quad=\frac{2}{b \sqrt{\pi}} \int_{0}^{1} \frac{d t}{(1-t)^{3 / 2} t^{1 / 2}} V\left(\epsilon, S, \check{\mathbf{X}}_{1}, \check{\mathbf{X}}_{2} ; \epsilon^{\prime}, S^{\prime}, \check{\mathbf{X}}_{1}^{\prime}, \check{\mathbf{X}}_{2}^{\prime}\right) .
\end{aligned}
$$

Mutatis mutandis we apply the integral transformation directly to the generating matrix elements $\mathcal{V}_{\left(l_{1} l_{2}\right) L M ;\left(l_{1}^{\prime} l_{2}^{\prime}\right) L M}\left(\epsilon, S ; \epsilon^{\prime}, S^{\prime}\right)$, 


$$
\begin{aligned}
& \mathcal{V}_{\left(l_{1} l_{2}\right) L M ;\left(l_{1}^{\prime} l_{2}^{\prime}\right) L M}^{C}\left(\epsilon, S ; \epsilon^{\prime}, S^{\prime}\right) \\
& =\frac{2}{\sqrt{\pi}} \int_{0}^{\infty} d x \mathcal{V}_{\left(l_{1} l_{2}\right) L M ;\left(l_{1}^{\prime} l_{2}^{\prime}\right) L M}\left(\epsilon, S ; \epsilon^{\prime}, S^{\prime}\right) \\
& \quad=\frac{2}{b \sqrt{\pi}} \int_{0}^{1} \frac{d t}{(1-t)^{3 / 2} t^{1 / 2}} \mathcal{V}_{\left(l_{1} l_{2}\right) L M ;\left(l_{1}^{\prime} l_{2}^{\prime}\right) L M}\left(\epsilon, S ; \epsilon^{\prime}, S^{\prime}\right)
\end{aligned}
$$

This, however, leads to a very intricate evaluation of the integral, and thus for the reduced generating matrix element of the Coulomb potential.

A better procedure is to generate the quantum numbers $K$ and $K^{\prime}$ first, by differentiation of $\mathcal{V}_{\left(l_{1} l_{2}\right) L M ;\left(l_{1}^{\prime} l_{2}^{\prime}\right) L M}\left(\epsilon, S ; \epsilon^{\prime}, S^{\prime}\right)$ on $S$ and $S^{\prime}$, then setting $S$ $=S^{\prime}=0$. This leads to reduced generating functions for each $K$ and $K^{\prime}$ now only dependent on $\epsilon$ and $\epsilon^{\prime}$, which are of a much simpler form and allow for an analytic integration in Eq. (102). The further derivation on matrix elements for $n$ and $n^{\prime}$ is then straightforward.

\section{CONCLUSION}

In this paper we presented a framework for a microscopic three-cluster model within the algebraic model for scattering.
It was shown that it is possible to obtain matrix elements for fully antisymmetrized three-cluster configurations, as well as a proper description for the three-cluster continuum in terms of a hyperspherical description. The corresponding AM equations in a multichannel description were also introduced. In the current work the individual clusters were limited to contain only $s$ orbitals, thus reducing the mass of clusters to that of a four-nucleon system. The latter restriction is, however, not a fundamental one, and was taken to restrict the analytical and calculational burden.

In order to prove the validity and feasibility of the current model we will apply it to two specific three-cluster configurations believed to be of importance to astrophysics, $\alpha+n$ $+n$ for ${ }^{6} \mathrm{He}$, and $\alpha+p+p$ for ${ }^{6} \mathrm{Be}$. These results appear in part II of this paper.

\section{ACKNOWLEDGMENTS}

This work was partially supported by an INTAS Grant ("INTAS-93-755-extension'"). One of the authors (V.V.) gratefully acknowledges the University of Antwerp (RUCA) for a "RAFO-gastprofessoraat 1998-1999' and the kind hospitality of the members of the research group Computational Quantum Physics of the Department of Mathematics and Computer Sciences, University of Antwerp, RUCA, Belgium.
[1] G. F. Filippov and I. P. Okhrimenko, Yad. Fiz. 32, 932 (1980) [Sov. J. Nucl. Phys. 32, 480 (1980)].

[2] G. F. Filippov, Yad. Fiz. 33, 928 (1981) [Sov. J. Nucl. Phys. 33, 488 (1981)].

[3] V. S. Vasilevsky and I. Yu. Rybkin, Yad. Fiz. 50, 662 (1989) [Sov. J. Nucl. Phys. 50, 411 (1989)].

[4] V. S. Vasilevsky, I. Yu. Rybkin, and G. F. Filippov, Yad. Fiz. 51, 112 (1990) [Sov. J. Nucl. Phys. 51, 71 (1990)].

[5] G. F. Filippov, V. S. Vasilevsky, and L. L. Chopovsky, Fiz. Elem. Chastits At. Yadra 16, 349 (1985) [Sov. J. Part. Nucl. 16, 153 (1985)].

[6] G. F. Filippov, S. P. Kruchinin, V. S. Vasilevsky, and L. L. Chopovsky, Yad. Fiz. 43, 843 (1986) [Sov. J. Nucl. Phys. 43, 536 (1986)].

[7] G. F. Filippov, V. S. Vasilevsky, and A. V. Nesterov, Nucl. Phys. A426, 327 (1984).

[8] G. F. Filippov, V. S. Vasilevsky, and A. V. Nesterov, Yad. Fiz. 40, 1418 (1984) [Sov. J. Nucl. Phys. 40, 901 (1984)].

[9] F. Arickx, P. Van Leuven, V. S. Vasilevsky, and A. V. Nesterov, in New Perspectives in Nuclear Structure, Proceedings of the 5th International Spring Seminar in Nuclear Physics, Ravello, Italy, 1995, edited by A. Covello (World Scientific, Singapore, 1996), p. 111.

[10] V. S. Vasilevsky, A. V. Nesterov, F. Arickx, and P. Van Leuven, Yad. Fiz. 60, 413 (1997) [Phys. At. Nucl. 60, 343 (1997)].

[11] G. F. Filippov, A. V. Nesterov, I. Yu. Rybkin, and S. V. Korennov, Fiz. El. Chastits At. Yadra 25, 1347 (1994) [Phys. Part. Nuclei 25, 569 (1994)].

[12] E. J. Heller and H. A. Yamani, Phys. Rev. A 9, 1201 (1974).
[13] E. J. Heller and H. A. Yamani, Phys. Rev. A 9, 1209 (1974).

[14] Y. I. Nechaev and Y. F. Smirnov, Yad. Fiz. 35, 1385 (1982) [Sov. J. Nucl. Phys. 35, 808 (1982)].

[15] P. Descouvemont, Phys. Rev. C 50, 2635 (1994).

[16] Y. Suzuki, K. Arai, Y. Ogawa, and K. Varga, Phys. Rev. C 54, 132 (1996).

[17] A. Csoto and K. Langanke, Nucl. Phys. A646, 387 (1999).

[18] G. F. Filippov, V. S. Vasilevsky, and L. L. Chopovsky, Yad. Fiz. 15, 1082 (1972) [Sov. J. Nucl. Phys. 15, 601 (1984)].

[19] G. F. Filippov, Riv. Nuovo Cimento 12, 1 (1989).

[20] V. S. Vasilevsky and F. Arickx, Phys. Rev. A 55, 265 (1997).

[21] Yu. A. Simonov, Yad. Fiz. 7, 722 (1968) [Sov. J. Nucl. Phys. 7, 1210 (1968)].

[22] M. Fabre de la Ripelle, Few-Body Syst. 14, 1 (1993).

[23] M. V. Zhukov, B. V. Danilin, D. V. Fedorov, J. M. Bang, I. J. Tompson, and J. S. Vaagen, Phys. Rep. 231, 151 (1993).

[24] F. Zernike and H. C. Brinkman, Proc. K. Ned. Akad. Wet. 33, 3 (1935).

[25] A. V. Nesterov, I. F. Gutich, and I. P. Okhrimenko, Yad. Fiz. 50, 19 (1989) [Sov. J. Nucl. Phys. 50, 12 (1989)].

[26] T. Ya. Mekhalashvili, Yu. F. Smirnov, and A. M. Shirokov, Yad. Fiz. 48, 1621 (1988) [Sov. J. Nucl. Phys. 48, 969 (1988)].

[27] H. A. Yamani and L. Fishman, J. Math. Phys. 16, 410 (1975).

[28] A. S. Jensen, D. V. Fedorov, and K. Riisager, Phys. Rev. C 49, 201 (1994).

[29] L. D. Landau and E. M. Lifshitz, Quantum Mechanics: Nonrelativistic Theory (Pergamon, Oxford, 1977).

[30] F. Calogero, Variable Phase Approach to Potential Scattering (Academic, New York, 1967). 
[31] V. V. Babikov, Phase Function Method in Quantum Mechanics (Nauka, Moscow, 1968).

[32] A. M. Perelomov, Commun. Math. Phys. 26, 222 (1972).

[33] A. M. Perelomov, Generalized Coherent States and Their Applications (Springer, Berlin, 1987).

[34] F. Arickx, J. Broeckhove, P. Van Leuven, V. Vasilevsky, and G. Filippov, Am. J. Phys. 62, 362 (1994).
[35] V. S. Vasilevsky, G. F. Filippov, F. Arickx, J. Broeckhove, and P. Van Leuven, J. Phys. G 18, 1227 (1992).

[36] J. Raynal and J. Revai, Nuovo Cimento A 68, 612 (1970).

[37] D. A. Varshalovich, A. N. Moskalev, and V. K. Khersonskii, Quantum Theory of Angular Momentum (World Scientific, Singapore, 1988). 